This item was submitted to Loughborough's Research Repository by the author.

Items in Figshare are protected by copyright, with all rights reserved, unless otherwise indicated.

\title{
A framework for design and optimization of tapered composite structures. Part II: Enhanced design framework with a global blending model
}

PLEASE CITE THE PUBLISHED VERSION

https://doi.org/10.1016/j.compstruct.2017.11.062

\section{PUBLISHER}

(C) Elsevier

VERSION

AM (Accepted Manuscript)

\section{PUBLISHER STATEMENT}

This work is made available according to the conditions of the Creative Commons Attribution-NonCommercialNoDerivatives 4.0 International (CC BY-NC-ND 4.0) licence. Full details of this licence are available at: https://creativecommons.org/licenses/by-nc-nd/4.0/

\section{LICENCE}

CC BY-NC-ND 4.0

\section{REPOSITORY RECORD}

Jing, Zhao, Qin Sun, Jian Q. Chen, and Vadim V. Silberschmidt. 2019. "A Framework for Design and Optimization of Tapered Composite Structures. Part II: Enhanced Design Framework with a Global Blending Model". figshare. https://hdl.handle.net/2134/28342. 


\title{
A framework for design and optimization of tapered composite structures. Part II: Enhanced design framework with a global blending model
}

\author{
Zhao Jing $^{\mathrm{a}, \mathrm{b}, *}$, Qin Sun ${ }^{\mathrm{a}, *}$, Jian Qiao Chen ${ }^{\mathrm{b}, *}$, Vadim V. Silberschmidt ${ }^{\mathrm{c}}$ \\ a School of Aeronautics, Northwestern Polytechnical University, Xi'an 710072, Shaanxi, China \\ b Department of Mechanics, Huazhong University of Science and Technology, Wuhan 430074, Hubei, China \\ ${ }^{c}$ Wolfson School of Mechanical, Electrical and Manufacturing Engineering, Loughborough University, LE11 3TU Leicestershire, UK
}

\section{A B S T R A C T}

\begin{abstract}
A high-dimensional variable design space in optimization problems for tapered composite structures presupposes a development of efficient computational techniques to improve the design efficiency and flexibility. In this work, a mathematical model for optimization of tapered composite structures with buckling and manufacturing constraints is developed, then a ply-drop-based global blending model (GBM) is suggested to address the layers' addition/deletion and blending problems. Within the framework proposed in Part I of this work (Compos Struct, 2016, 154: 106-128), by incorporating the global blending model, operations of add-layer, exchange-blend and sequence-adjustment are revised and enhanced. The GBM can not only characterize the global blending property, but also guide the maximum blending design. Accordingly, the design framework is simplified, and its computational cost is reduced significantly since there is no iteration of shape prediction and stacking-sequence optimization procedure. An 18-panel benchmark problem is adopted to verify the enhanced design framework, as compared with previous design results, the obtained better solution with higher efficiency implies its feasibility and potential for effective design of tapered composite structures.
\end{abstract}

\author{
Keywords: \\ Tapered composite structures \\ Optimization \\ Global blending model \\ Stacking sequence \\ Buckling \\ Manufacturing constraints
}

\section{Introduction}

Over the last two decades, application of composite structures in engineering has become crucial for many engineering sectors thanks to their high mechanical performance and reduction of manufacturing cost. Development of numerical simulation techniques [1] and computational optimization design methods [2] underpinned wide and deep analysis of composites by scientists and researchers. Design of composite structures with variable stiffness is a particularly difficult problem due to their high-dimensional design spaces with different type of variables. To reduce the design space, composite structures are often divided into panels or regions [3]. The design process is moving from a global structural level to a local (panel) level for varying loads transferring between parts. However, structural integrity is not only important for load transition between adjacent zones, but also the main concern for manufacturing. So, a blending problem for variable-thickness panels is important for design of tapered composite structures [4]. The blending requirement is a critical constraint for stacking sequence design, since the stacking sequences of two adjacent laminates are independent variables. A challenging work is to achieve a minimumweight blended composite structure with an optimal stacking sequence involving complex manufacturing constraints.

This challenging problem attracted many researchers. Efficient optimization techniques have been developed for various composite components, e.g. a wing box $[3,6,7]$, blade stiffened composite panels [5,15], an 18-panel horseshoe [8-12,23-27], a wing skin structure $[19,20]$, a sailing boat [28], a vertical tail [29], a horizontal stabilizer of F-16A/B [30], an aircraft fuselage [32], etc. Various heuristic algorithms were adopted to deal with this problem, such as an improving hit-and-run (IHR) algorithm [2], a genetic algorithm (GA) [3-12,24,25,27,30,31], an ant-colony optimisation (ACO) [14], an evolutionary algorithm (EA) $[26,28,29]$, etc. Specific computational techniques were developed for stacking-sequence design, especially focusing on the blending issue: various parameterization techniques $[13,15,28,29]$, a backtracking method [16-18], an outer approximation method for stacking-sequence design coupled with a level set method for layer-shape optimization [32], a global shared-layer blending (GSLB) method [20], a permutation search (PS) [21], and a sequential permutation table method (SPT) [22], etc.

In order to achieve a blended structure, blending definitions were proposed to characterize a continuity property of layers between adjacent laminates as well as guide the blending design. In 2001, the term

\footnotetext{
* Corresponding authors at: School of Aeronautics, Northwestern Polytechnical University, Xi'an 710072, Shaanxi, China (Z. Jing and Q. Sun).

E-mail address: jingzhao@hust.edu.cn (Z. Jing).
} 


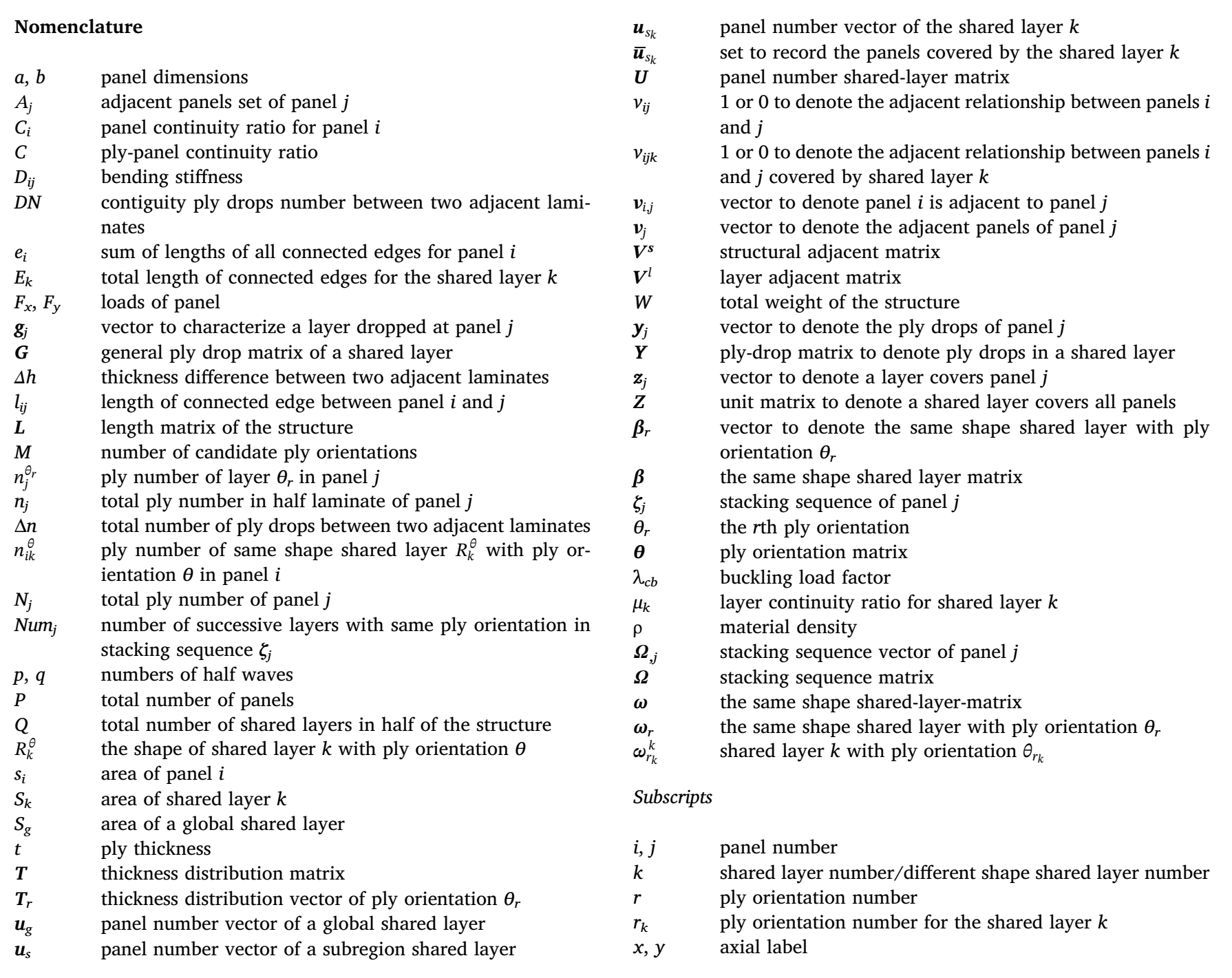

"blending" [2] was employed to ensure a capability for manufacturing of multi-panel composite structures. In the same year, two continuity measures were defined based on material composition and a stacking sequence [3]. Later, a guide-based design concept was proposed with inwardly and outwardly blending to maintain structural integrity [9]. Besides, a general blending definition was proposed for two adjacent laminates [4]. Particularly, a patch concept and a graph-based parameterization concept are introduced in [28] and [29], respectively. Apart from the above blending definitions, a manufacturing-cost design concept considering manufacturing time and labour is introduced in [30], with a cutting length and stacking area of layers in a tapered composite structure being used as index to reduce number of prepregs.

The above blending definitions mainly characterize the blending property between two adjacent laminates and, then, by extending the layers to the overall structure, as a result, ensuring the blending requirement and manufacturability. However, it is difficult for them to characterize the overall blending property, since they are used as a concept in the design process or as constraints in the objective function, making them hard to guide the detailed blending design procedures. Instinctually, ply drops are caused by thickness difference of adjacent laminates, and further sequence design and manufacturing constraints result in ply-orientation mismatches or stacking position mismatches, leading to an increase of ply drops. The blending property is also related to a weight penalty, the better the blending property the larger the weight [3]. As expected, the definition of blending should not only consider the blending property between two adjacent laminates, but also a thickness distribution and a layer shape, which can characterize the overall blending property.

In Part I of this work [23], a framework for design of tapered composite structures was developed in terms of decomposition of design variables, in which a concept that extending layers from individual panels to the overall structure is used to improve the blending property. The design process was divided into global and local levels. At the former level, a minimum thickness distribution, shapes of layers, ply orientations of layers and an optimal stacking sequence were identified; at the latter level, several design techniques were developed to improve the blending property of the solution. Unfortunately, the blending property of the design results cannot be assessed at the local level, making some of the design process complex and hard to apprehend. In this work, to assess the blending property and guide the design process, a ply-drop-based global blending model (denoted as GBM) is proposed. A key parameter - ply-panel continuity ratio is introduced as an overall blending index, it is an average of panel continuity ratios (individual panel blending index), in which thickness variation, ply drops and a layer shape are accounted for. This model integrates the global blending property and the manufacturing cost design concept [30] since the cutting length of layers (ply drops) is included.

Generally, an optimal solution is a trade-off of weight, manufacturing constraints and blending properties to satisfy mechanical requirements (see Fig. 1). Design stages often change from individual 


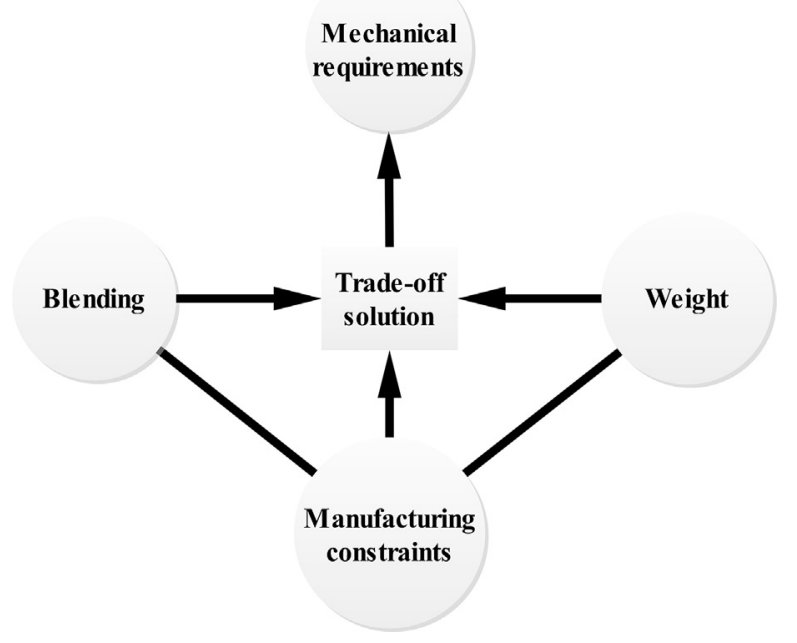

Fig. 1. Trade-off solution for optimization of tapered composite structures.

panels to a global structural level $[2,23]$. In this work, the GBM is applied to the design of an 18-panel problem presented in [23], especially at the local level within the add-layer and exchange-blend operations. By taking advantage of the GBM, the layer shape prediction and stacking sequence optimization procedures are performed only once since the GBM can identify exact positions of added/deleted layers and guide the exchange-blend operation of different ply orientations for maximizing the blending property. As a result, the design process is simplified and the computational cost is reduced.

The remainder of this article is arranged as follows. In Section 2, a mathematical model for optimization of tapered composite structures with buckling and manufacturing constraints is constructed. Based on the mathematical model, the definition of a layer continuity ratio, connected edges, a panel continuity ratio and a ply-panel continuity ratio are introduced to construct the global blending model in Section 3. Subsequently, in Section 4, the add-layer, exchange-blend and sequence-adjustment operations are revised and enhanced based on the global blending model. Then, the original design framework is simplified and rebuilt in Section 5. After that, the benchmark 18-panel problem is optimized by employing the enhanced design framework in Section 6. Finally, conclusions drawn from the present work are summarized in Section 7.

\section{Development of design model for tapered composite structures}

In this section, the mathematical model of adjacent relationship between panels, shared layers, stacking sequence matrix and thickness distribution are constructed. Afterwards, buckling and manufacturing constraints are introduced. Finally, the objective function of the tapered composite structures optimization problem is presented. The purpose of this section is to make preparations for the development of global blending model in Section 3.

\subsection{Mathematical model}

\subsubsection{Characterization of adjacent relationship between panels}

To elucidate the definition of blending parameters in tapered composite structures, a 9-panel tapered composite structure with each panel in unit size (both length and width are equal to 1 ) and numbered from 1 to 9 (see Fig. 2(a)) is used for demonstration of different parameters. The thickness distribution is given in Fig. 2(b). The total number of panels is $P$, here $P=9$.

For a panel $j(j=1,2, \ldots, P)$, if panel $i(i \neq j, i=1,2, \ldots, P)$ is adjacent to it, a vector $\boldsymbol{v}_{i, j}$ is defined to characterize the adjacent relationship:

$\boldsymbol{v}_{i, j}=[\underbrace{\overbrace{0,0, \ldots 0}^{i-1}, 1,0, \ldots, 0}_{P}]^{T}$.

Assume that the adjacent panels of panel $j$ are in a set $A_{j}$, $A_{j}=\{i$ panel $i$ is adjacent to panel $j\}$, the adjacent panels vector of panel $j$ is defined as:

$\boldsymbol{v}_{j}=\sum_{i}^{i \in A_{j}} \boldsymbol{v}_{i, j}$.

Thus, the structural adjacent matrix $\boldsymbol{V}^{s}$ can be obtained:

$\boldsymbol{V}^{S}=\left[\boldsymbol{v}_{1} \boldsymbol{v}_{2} \cdots \boldsymbol{v}_{P}\right]$

Since two adjacent panels $i$ and $j$ have symmetrical relationship for the adjacent property, $\boldsymbol{V}^{\boldsymbol{s}}$ is a symmetric matrix. Accordingly, the relationship of every two adjacent panels of the 9-panel structure can be described by the structural adjacent matrix [21]:

$\boldsymbol{V}^{s}=\left[\begin{array}{ccccccccc}0 & v_{12} & 0 & v_{14} & 0 & 0 & 0 & 0 & 0 \\ v_{21} & 0 & v_{23} & 0 & v_{25} & 0 & 0 & 0 & 0 \\ 0 & v_{32} & 0 & 0 & 0 & v_{36} & 0 & 0 & 0 \\ v_{41} & 0 & 0 & 0 & v_{45} & 0 & v_{47} & 0 & 0 \\ 0 & v_{52} & 0 & v_{54} & 0 & v_{56} & 0 & v_{58} & 0 \\ 0 & 0 & v_{63} & 0 & v_{65} & 0 & 0 & 0 & v_{69} \\ 0 & 0 & 0 & v_{74} & 0 & 0 & 0 & v_{78} & 0 \\ 0 & 0 & 0 & 0 & v_{85} & 0 & v_{87} & 0 & v_{89} \\ 0 & 0 & 0 & 0 & 0 & v_{96} & 0 & v_{98} & 0\end{array}\right]$,

the elements $v_{i j}(i, j=1,2, \ldots, P)$ are equal to 1 or 0 . If panel $i$ is adjacent to panel $j, v_{i j}=1$; otherwise, $v_{i j}=0$. For the 9-panel structure in Fig. 2, non-zero elements $v_{i j}$ in Eq. (4) are equal to 1.

Three kinds of layers are defined in a tapered composite structure, as shown in Fig. 3:

A global shared layer - a layer covers all panels.

A subregion shared layer - a layer covers not all panels.

An individual layer - a layer covers only one panel.

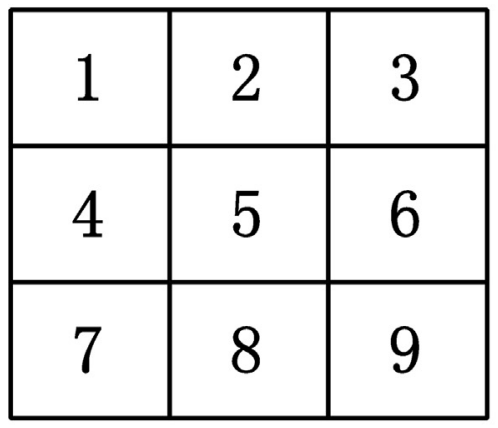

(a)

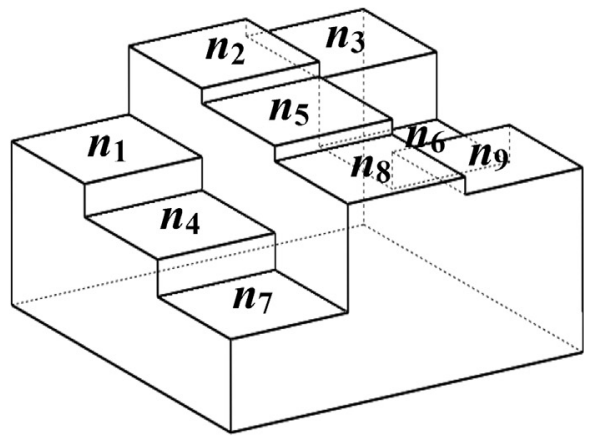

(b)

Fig. 2. Tapered composite structure: (a) 9-panel structure, (b) variable thickness $n_{\mathrm{i}}$ of 9 panels. 
Global shared layer

Subregion shared layer

Individual layer

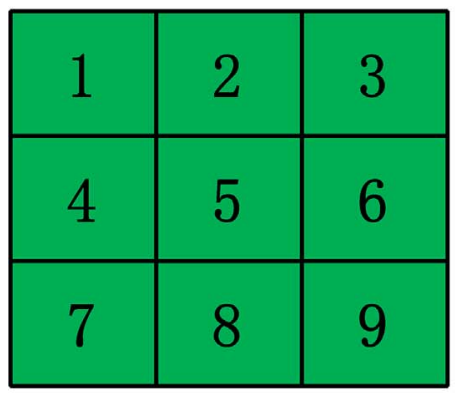

$\bar{u}=\{1,2,3,4,5,6,7,8,9\}$

(a)

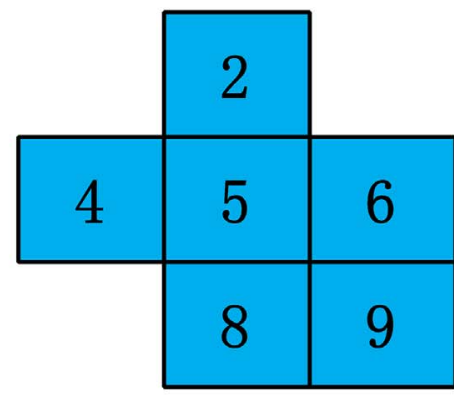

$\bar{u}=\{2,4,5,6,8,9\}$

(b)
Fig. 3. Three kinds of layers: (a) global shared layer, (b) subregion shared layer, (c) individual layer.
An individual layer is a special case of subregion shared layer.

If a shared layer dropped at panel $j$, according to the definition in Eqs. (1)-(3), for any panel $i$ adjacent to panel $j$, the vectors $\boldsymbol{v}_{i, j}=\mathbf{0}$, thus, in the adjacent matrix of the shared layer the corresponding $j$ th row and jth column are $\mathbf{0}$. A tapered composite structure consisting of shared layers with various shapes. The adjacent matrix of a shared layer is determined by the panels it covers, hence, the adjacent matrices of different shape layers are different. Therefore, a three dimensional layer adjacent matrix $\boldsymbol{V}_{P \times P \times Q}^{l}$ is defined to characterize the adjacent relationship of panels in different shared layers, and assume that there are $Q$ shared layers:

$$
\begin{aligned}
\boldsymbol{Z} & =\left[\begin{array}{cccc}
1 & 0 & \cdots & 0 \\
0 & 1 & \ddots & \vdots \\
\vdots & \ddots & \ddots & 0 \\
0 & \cdots & 0 & 1
\end{array}\right]_{P \times P}=\left[\boldsymbol{z}_{1}, \ldots, \boldsymbol{z}_{j}, \ldots, \boldsymbol{z}_{P}\right], \quad \boldsymbol{z}_{j}=[\overbrace{0, \cdots, 0,1,0, \cdots, 0}^{j-1}]^{\mathrm{T}}, \quad j \\
& =1,2, \ldots, P
\end{aligned}
$$

where the column vector $\boldsymbol{z}_{j}$ denotes the $j$ th panel is covered by the shared layer. To define the ply drops of a shared layer, another ply-drop matrix $\boldsymbol{Y}_{P \times P}$ is defined:

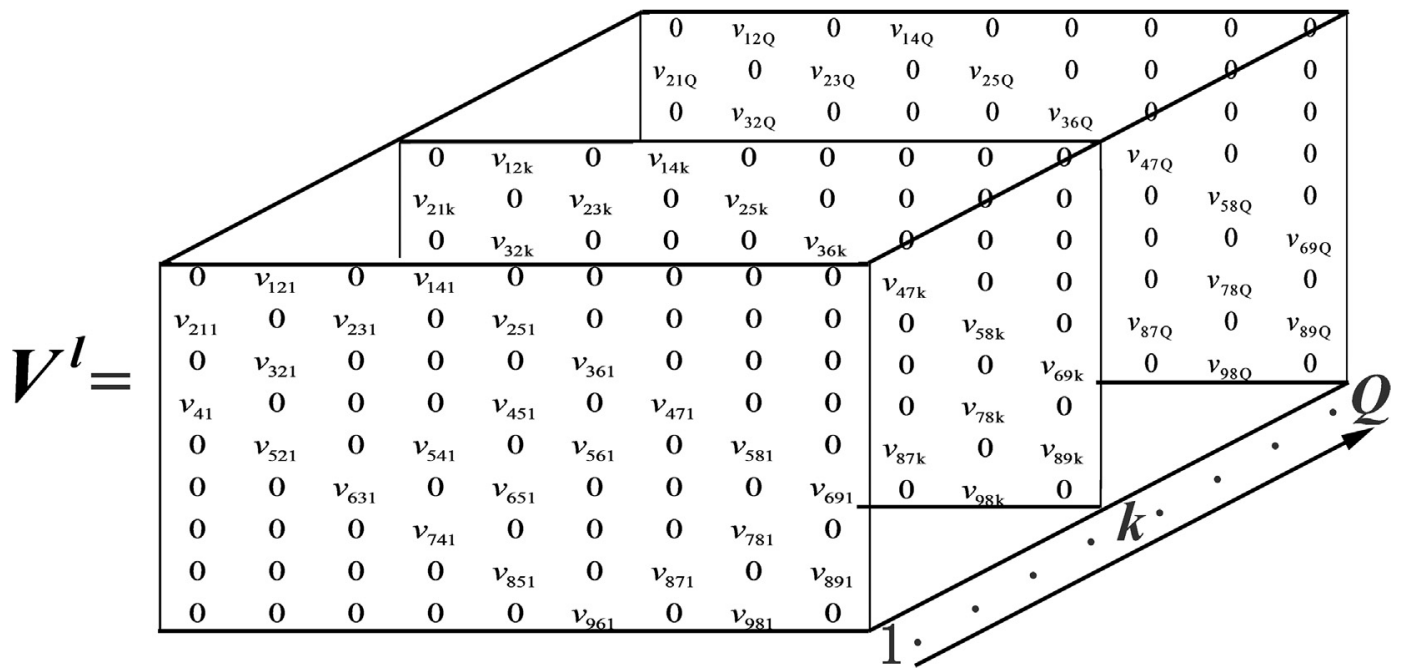

where the shared layer number $k$ varies from 1 to $Q$, and the elements $v_{i j k}$ represents the adjacent relationship between panels $i$ and $j$ covered by the shared layer $k$, which is 1 or 0 .

\subsubsection{Modelling of the shared layers}

Considering symmetrical and balanced laminates in a tapered composite structure. First, a shared layer that covers all panels in the structure is defined by a unit matrix $\boldsymbol{Z}_{P \times P}$ :

$$
\begin{aligned}
\boldsymbol{Y} & =\left[\begin{array}{cccc}
0 & 0 & \cdots & 0 \\
0 & -1 & \ddots & \vdots \\
\vdots & \ddots & \ddots & 0 \\
0 & \cdots & 0 & 0
\end{array}\right]_{P \times P}=\left[\boldsymbol{y}_{1}, \ldots, \boldsymbol{y}_{j}, \ldots, \boldsymbol{y}_{P}\right], \boldsymbol{y}_{j}=[\overbrace{0, \cdots, 0,-1,0, \cdots, 0]^{\mathrm{T}}, \quad j}^{j-1} \\
& =1,2, \ldots, P,
\end{aligned}
$$

where the column vector $\boldsymbol{y}_{j}$ denotes that the layer at $j$ th panel is dropped in the shared layer, which is represented by -1 in $\boldsymbol{y}_{j}$. Combining Eqs. (6) and (7), a general ply drop matrix $\boldsymbol{G}_{P \times P}$ is defined with its column vector $\boldsymbol{g}_{j}$ to describe the panels covered by a shared layer: 
$\boldsymbol{G}=\boldsymbol{Z}+\boldsymbol{Y}, \boldsymbol{G} \neq \mathbf{0}$

$\mathbf{g}_{j}=\boldsymbol{z}_{j}+\boldsymbol{y}_{j}, \quad j=1,2, \ldots, P$

For the column vector $\boldsymbol{g}_{j}$

$\left|g_{j}\right|=\left\{\begin{array}{l}1, \text { the layer covers panel } j \\ 0, \text { the layer drops at panel } j\end{array}\right.$

where the general ply drop matrix $G$ is a diagonal matrix with only 0 or 1. $\boldsymbol{G} \neq \mathbf{0}$ means that the shared layer cannot drop at all panels (otherwise the shared layer is non-existence). $\boldsymbol{G}=\boldsymbol{I}(\boldsymbol{Z}=\boldsymbol{I}, \boldsymbol{Y}=\mathbf{0})$ denotes a global shared layer without any ply drop.

Define a panel number vector $\boldsymbol{u}_{g}$

$\boldsymbol{u}_{g}=[1,2,3, \ldots, P]$

It represents the panels covered by a global shared layer ( $P$ is the total number of panels). Thus the panels covered by a subregion shared layer is

$\boldsymbol{u}_{s}=\boldsymbol{u}_{g} \boldsymbol{G}$

The $j$ th element 0 in $\boldsymbol{u}_{s}$ denotes that the shared layer is dropped at panel $j$. Suppose that there are $Q$ shared layers in a tapered composite structure, a panel number shared-layer matrix $\boldsymbol{U}_{Q \times P}$ with its row vectors $\boldsymbol{u}_{s_{k}}$ are defined to characterize the panels covered by shared layers:

$\boldsymbol{U}=\left[\begin{array}{c}\boldsymbol{u}_{s_{1}} \\ \vdots \\ \boldsymbol{u}_{s_{k}} \\ \vdots \\ \boldsymbol{u}_{s_{Q}}\end{array}\right]_{Q \times P}, \quad \boldsymbol{u}_{s_{k}}=[1,2, \ldots, j, \ldots, P], \quad j=0,1, \ldots, P ; \quad k=1,2, \ldots, Q$

where $\boldsymbol{u}_{s_{k}}$ denotes the panels covered by the $k$ th shared layer as defined in Eq. (11). Thus, the panels covered by shared layer $k$ is expressed in a set:

$\overline{\boldsymbol{u}}_{s_{k}}=\left\{\boldsymbol{u}_{s_{k}}\left(\boldsymbol{u}_{s_{k}} \neq 0\right)\right\}, k=1,2, \ldots, Q$

The new set $\overline{\boldsymbol{u}}_{s_{k}}$ contains all non-zero elements in vector $\boldsymbol{u}_{s_{k}}$. The set $\overline{\boldsymbol{u}}_{s_{k}}$ is defined to characterize the panels covered by the shared layer $k$, as shown in Fig. 3.

Assume that there are $M$ candidate ply orientations $\left\{\theta_{1}, \theta_{2}, \ldots, \theta_{\mathrm{M}}\right\}$, since each shared layer has $M$ choices of ply orientations, a ply orientation matrix $\boldsymbol{\theta}_{M \times P}$ is defined as:

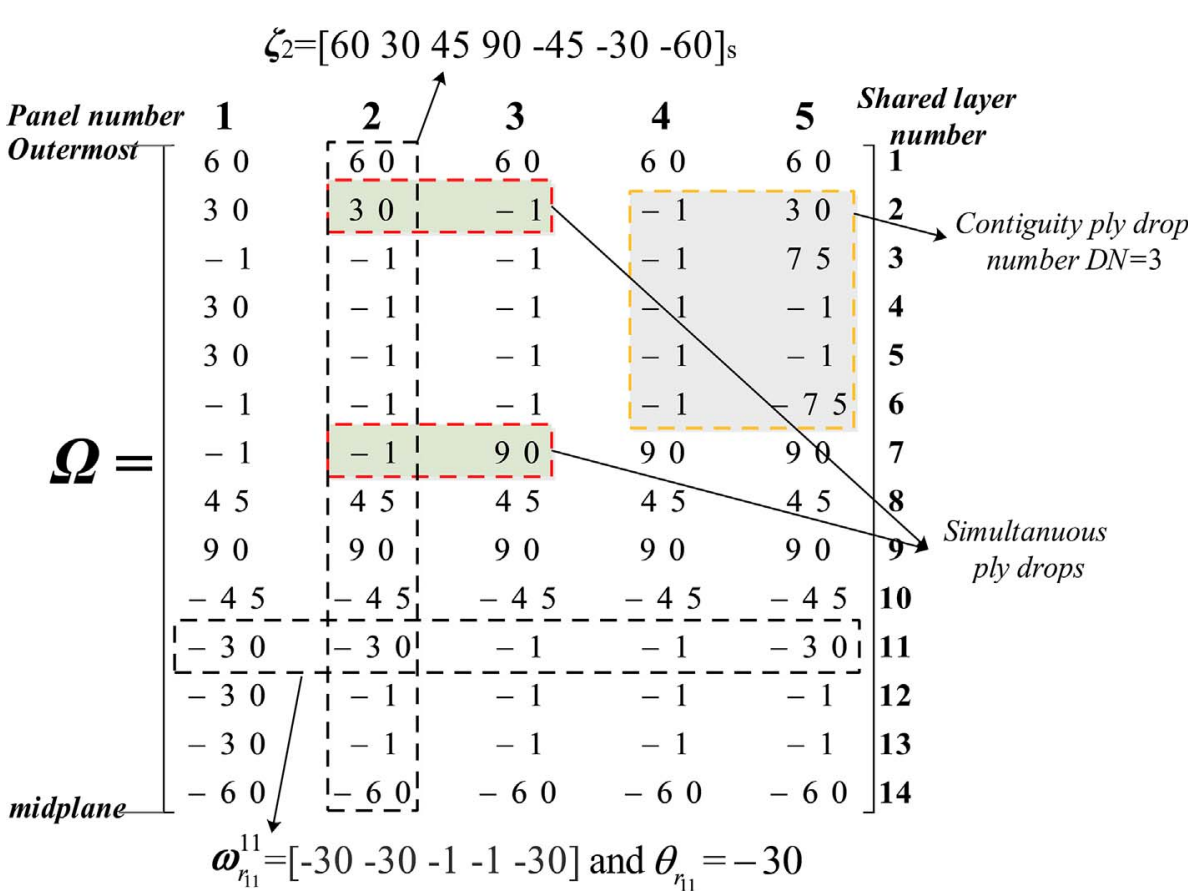

$\theta=\left[\begin{array}{cccc}\theta_{1} & \theta_{1} & \cdots & \theta_{1} \\ \theta_{2} & \theta_{2} & \cdots & \theta_{2} \\ \vdots & \vdots & \ddots & \vdots \\ \theta_{M} & \theta_{M} & \cdots & \theta_{M}\end{array}\right]_{M \times P}$

For each shared layer, defining a same shape shared-layer-matrix $\omega_{M \times P}$ with its row vector $\omega_{r}$ :

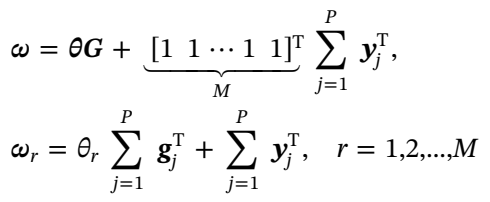

where the row vector $\omega_{r}$ is the shared layer with ply orientation $\theta_{r}$ $(r=1,2, \ldots, M)$, and element $\omega_{r j}=-1,(j=1,2, \ldots, P)$ denotes that the shared layer drops at panel $j$. The shapes of shared layer $\omega_{r}$ are controlled by the variation of general ply drop matrix $\boldsymbol{G}$. Assume that there are $Q$ shared layers with some different shapes, the $k$ th $(k=1,2, \ldots, Q)$ shared layer is $\omega_{r_{k}}^{k}$ with ply orientation $\theta_{r_{k}}$ and general ply drop matrix $\boldsymbol{G}^{k}$ (corresponding $\boldsymbol{g}^{k}$ and $\boldsymbol{y}^{k}$ ), thus a shared layer is expressed as:

$\omega_{r_{k}}^{k}=\theta_{r_{k}} \sum_{j=1}^{P}\left(\mathbf{g}_{j}^{k}\right)^{\mathrm{T}}+\sum_{j=1}^{P}\left(\boldsymbol{y}_{j}^{k}\right)^{\mathrm{T}}, \quad r_{k}=1,2, \ldots, M ; \quad k=1,2, \ldots Q$

in the shared layer $\omega_{r_{k}}^{k}$, the variation of shape is characterized by $\mathbf{g}_{j}^{k}$ and $\boldsymbol{y}_{j}^{k}$ and the variation of ply orientation is characterized by $\theta_{r_{k}}$.

\subsubsection{Stacking sequence matrix}

Since there are $Q$ shared layers with some different shapes and the $k$ th $(k=1,2, \ldots, Q)$ shared layer is $\omega_{r_{k}}^{k}$ with ply orientation $\theta_{r_{k}}$, the stacking sequence matrix $\boldsymbol{\Omega}_{Q \times P}$ of a tapered composite structure is a permutation of the shared layers $\omega_{r_{k}}^{k}$ :

$\boldsymbol{\Omega}=\left[\begin{array}{c}\omega_{r_{1}}^{1} \\ \vdots \\ \omega_{r_{k}}^{k} \\ \vdots \\ \omega_{r_{Q}}^{Q}\end{array}\right]_{Q \times P}, \quad r_{k}=1,2, \ldots, M ; \quad k=1,2, \ldots, Q$,

in which variable stiffness of individual panels can be obtained by dividual panel stacking sequence, shared layers, contiguity ply drops and simultaneous ply drops. 
exchanging the sequence of rows in the stacking sequence matrix $\boldsymbol{\Omega}$. Note that, in this work, only symmetrical laminates are considered, hence $\boldsymbol{\Omega}$ is the stacking sequence matrix of a half of a tapered composite structure. The first row is defined as the outermost position and the last row is above the midplane. A simple example of the stacking sequence matrix is given in Fig. 4, which demonstrates the shared layers and the stacking sequences of individual panels. The contiguity and simultaneous ply drops are illustrated in the stacking sequence matrix in Fig. 4, which provides convenience for detection of ply drops in the structure.

In $\boldsymbol{\Omega}$, the column vector $\boldsymbol{\Omega}_{, j}$ is the stacking sequence of panel $j$, in which the element -1 means that there is no layer at this stacking position (ply drop), thus, the elements -1 should be deleted and the stacking sequence $\zeta_{j}$ of panel $j$ can be obtained

$\zeta_{j}=\left[\boldsymbol{\Omega}_{j}\left(\boldsymbol{\Omega}_{j} \neq-1\right)\right]^{\mathrm{T}}$.

The function $\boldsymbol{\Omega}_{, j}\left(\boldsymbol{\Omega}_{j} \neq-1\right)$ denotes that all " -1 " are deleted from the vector $\boldsymbol{\Omega}_{, j}$. As a result, a new vector $\zeta_{j}$ is obtained. In addition, detailed detection procedures of contiguity and simultaneous ply drops are introduced in the section of manufacturing constraints and the Appendix A.

\subsubsection{Thickness distribution}

By counting the ply number of different ply orientations in $\zeta_{j}$ $(j=1,2, \ldots, P)$, the thickness distribution matrix $\boldsymbol{T}_{M \times P}$ is obtained (see Fig. 1(b)):

$\boldsymbol{T}=\left[\begin{array}{cccc}n_{1}^{\theta_{1}} & n_{2}^{\theta_{1}} & \cdots & n_{P}^{\theta_{1}} \\ n_{1}^{\theta_{2}} & n_{2}^{\theta_{2}} & \cdots & n_{P}^{\theta_{2}} \\ \vdots & \vdots & \ddots & \vdots \\ n_{1}^{\theta_{M}} & n_{2}^{\theta_{M}} & \cdots & n_{P}^{\theta_{M}}\end{array}\right]_{M \times P}$,

where $n_{j}^{\theta_{r}}$ is the ply number of layer $\theta_{r}$ in panel $j(r=1,2, \ldots, M ; j=1,2$, $\ldots, P$ ), the total ply number (in a half of the laminate) of panel $j$ is

$n_{j}=\sum_{r=1}^{M} n_{j}^{\theta_{r}}$.

And according to Eq. (19), the thickness distribution $\boldsymbol{T}_{r}$ of ply orientation $\theta_{r}$ is:

$\boldsymbol{T}_{r}=\left[n_{1}^{\theta_{r}} n_{2}^{\theta_{r}} \ldots n_{P}^{\theta_{r}}\right], \quad r=1,2, \ldots, M$

Eventually, for an optimization problem of a multi-panel tapered composite structure, the objective is to minimize the weight
$W=\sum_{j=1}^{P} \rho t s_{j} N_{j}$

where $P$ is the total number of panels, $\rho$ is the density, $t$ is the ply thickness, $s_{j}$ is the area of panel $j, N_{j}$ (even number) is the total number of plies of panel $j$ and $N_{j}=2 n_{j}$. Note that, in each panel of tapered composite structure the laminate is symmetrical and balanced.

\subsection{Buckling constraint}

The design objective for optimization of a tapered composite structure is minimizing the weight (Eq. (22)) while satisfying mechanical and manufacturing requirements. A tapered composite structure is often under axial compressive loading. To save weight, it is divided into individual panels with variable thickness. In each panel, buckling is one of the main critical failure modes. In this work, buckling of symmetrical and balanced laminates with even ply numbers, simply supported on boundaries, under in-plane compressive loads $\left(F_{\mathrm{x}}, F_{\mathrm{y}}\right)$ is considered. The buckling load factor is [23]:

$$
\begin{aligned}
\lambda_{c b}= & \pi^{2}\left[D_{11}\left(\frac{p}{a}\right)^{4}+2\left(D_{12}+2 D_{66}\right)\left(\frac{p}{a}\right)^{2}\left(\frac{q}{b}\right)^{2}\right. \\
& \left.+D_{22}\left(\frac{q}{b}\right)^{4}\right]\left[F_{x}\left(\frac{p}{a}\right)^{2}+F_{y}\left(\frac{q}{b}\right)^{2}\right]^{-1}
\end{aligned}
$$

where $p$ and $q$ are the numbers of half-waves along the $x$ and $y$ directions respectively. $a$ and $b$ are the length and width of the panel respectively. $D_{11}, D_{12}, D_{22}$ and $D_{66}$ are the bending stiffness of the laminate ( $D_{16}$ and $D_{26}$ are neglected for an orthotropic plate), since they depend on the stacking sequence $\zeta_{j}$, the buckling constraint is

$\lambda_{c b}\left(\zeta_{j}\right) \geqslant 1, \quad j=1,2, \ldots, P$.

\subsection{Manufacturing constraints}

Based on the above mathematical model, the manufacturing constraints are considered for the optimization problem. Some new constraints for ply drops are added to improve the global blending property. Due to the complex of the manufacturing constraints and by taking advantage of the stacking sequence matrix, the procedures to detect different constraints are introduced in the Appendix A.

CT1.Symmetry. The stacking sequence $\zeta_{j}(j=1,2, \ldots, P)$ is symmetrical about the mid-plane.

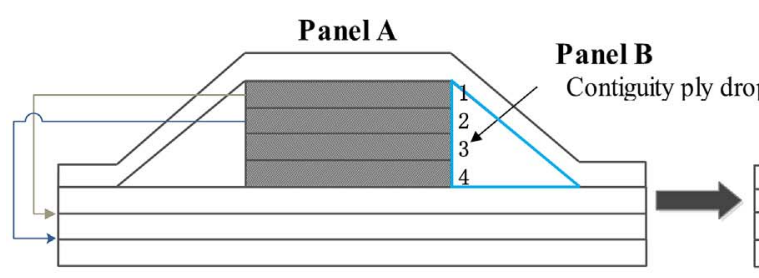

(a)

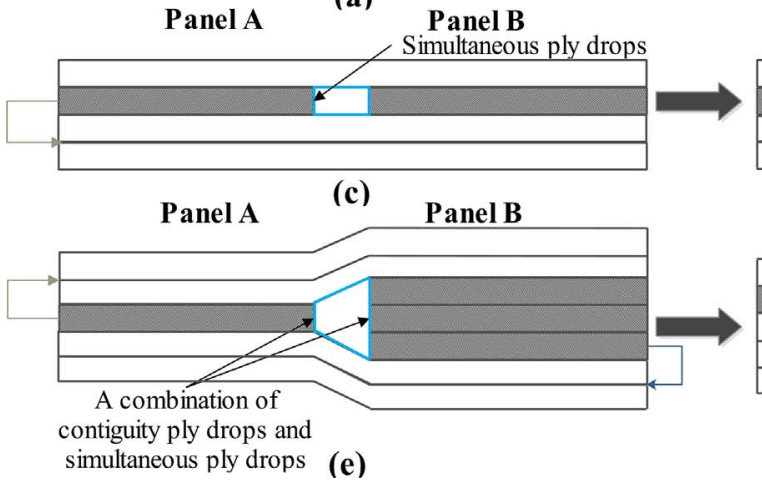

Panel A

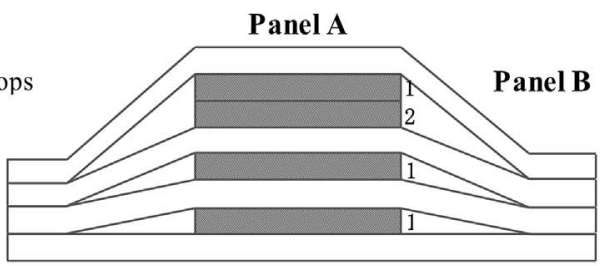

(b)

Panel A
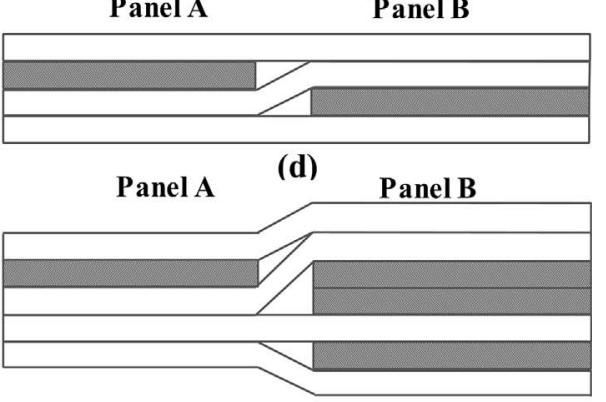

(f)
Fig. 5. Ply drop cases and measures to deal with them: (a) and (b) contiguity ply drops and after exchanging stacking positions; (c) and (d) simultaneous ply drops and after exchanging stacking positions; (e) and (f) a combination of contiguity ply drops and simultaneous ply drops, and after exchanging stacking positions. 
CT2.Balance. The number of ply orientations $+\theta$ and $-\theta$ should be the same in the stacking sequence $\zeta_{j}(j=1,2, \ldots, P)$.

CT3.Contiguity. No more than 3 layers with the same ply orientation stack together in the stacking sequence $\zeta_{j}(j=1,2, \ldots, P)$.

CT4. Disorientation. Difference between ply orientations of two consecutive plies should not exceed $45^{\circ}$ in the stacking sequence $\zeta_{j}$ $(j=1,2, \ldots, P)$.

CT5. Contiguity ply-drop number limit $D N \leq 2$. As shown in Fig. 5(a), there are 4 contiguity ply drops between two adjacent panels $\mathrm{A}$ and $\mathrm{B}$, the way to decrease $D N$ is to exchange the drop layers to other stacking positions, see Fig. 5(b). This constraint is only imposed between two adjacent panels.

CT6. Simultaneous ply-drop limit. No simultaneous ply drops are allowed in the structure (see Fig. 5(c)). Similarly, the drop layer is exchanged to the other stacking positions (see Fig. 5(d)). Moreover, more drop layers should be adjusted for a combination of contiguity ply drops and simultaneous ply drops (see Fig. 5(e) and (f)).

CT7. Ply orientation mismatch limit $\Delta n-\Delta h \leq 2$. Where $\Delta n$ and $\Delta h$ are the total ply drop numbers and the thickness difference between two adjacent panels, respectively. There should be no more than 2 ply orientation mismatches between adjacent laminates, the way to deal with this limit is to adjust the proportion of ply orientations in one laminate and blend with the shared layers of its adjacent laminates.

CT8. Covering. The covering layers on the lower and upper surfaces should not be dropped.

CT9. Continuity layers. All layers of the thinnest panel must cover the whole structure.

The objective of the tapered composite structures optimization problem is to minimize the weight (Eq. (22)) while satisfy the buckling constraint (Eq. (24)) and the above manufacturing constraints CT1-CT9.

\section{Global blending model (GBM)}

In order to save weight, tapered composite structures are divided into panels with variable thickness for varying loads, resulting in ply drops between adjacent laminates and, therefore forming a discontinuous and variable shape of layers in the structure. The layer shape is determined by its connectivity to the layer of its adjacent panels, which is controlled by thickness, ply orientations, stacking sequence and manufacturing constraints. The property of connectivity not only ensures a continuous load path, but also decreases a risk of stress concentration and delamination. Therefore, the connectivity represents the important part of blending property of laminated structures, and it should be considered in designing high performance composite structures.

On the other hand, from the manufacturing perspective, the fewer the shared layers and the larger the area of the shared layers, the fewer the stacked prepregs and the shorter the cutting length are required [30]. Therefore, to save the manufacturing cost, the global blending model proposed in this study also involves the effects of the cutting length and the area of layers.

\subsection{Global blending parameters}

\subsubsection{Layer continuity ratio}

The design of a tapered composite structure tries to extend each layer as much as possible to ensure its maximum blending property [2]. Thus, a bigger area of a layer results in a better blending property. The layer continuity ratio $\mu_{k}$ is defined as the area ratio of shared layer $k$ to the global shared layer:

$\mu_{k}=\frac{S_{k}}{S_{g}}$,

where $k(k=1,2, \ldots, Q)$ is the number of shared layer, $S_{k}$ is the area of the shared layer $k, S_{g}$ is the area of the global shared layer, and
$S_{k}=\sum_{i}^{i \in \overline{\boldsymbol{u}}_{S_{k}}} s_{i}$.

Here, $s_{i}$ is the area of the panel $i, \overline{\boldsymbol{u}}_{s_{k}}$ represents the panels covered by the shared layer $k$.

\subsubsection{Connected edges}

To evaluate the layer continuity ratio, ply drops of a shared layer should be identified since they are the boundaries of the layer. The connected edges are defined to characterize the ply drops. Definition of the connected edges for the 9-panel structure is given in Fig. 6: $l_{i j}$ is the length of the connected edge of panel $i$ and $j(i, j=1,2, \ldots, 9)$, the adjacent relationship of panel $i$ and $j$ is determined by $v_{i j}$ in Eq. (4), and $e_{i}$ is the sum of lengths of all connected edges for panel $i$. The definition of length matrix $\boldsymbol{L}$ of connected edge for the 9-panel structure is

$\boldsymbol{L}=\left[\begin{array}{ccccccccc}0 & l_{12} & 0 & l_{14} & 0 & 0 & 0 & 0 & 0 \\ l_{21} & 0 & l_{23} & 0 & l_{25} & 0 & 0 & 0 & 0 \\ 0 & l_{32} & 0 & 0 & 0 & l_{36} & 0 & 0 & 0 \\ l_{41} & 0 & 0 & 0 & l_{45} & 0 & l_{47} & 0 & 0 \\ 0 & l_{52} & 0 & l_{54} & 0 & l_{56} & 0 & l_{58} & 0 \\ 0 & 0 & l_{63} & 0 & l_{65} & 0 & 0 & 0 & l_{69} \\ 0 & 0 & 0 & l_{74} & 0 & 0 & 0 & l_{78} & 0 \\ 0 & 0 & 0 & 0 & l_{85} & 0 & l_{87} & 0 & l_{89} \\ 0 & 0 & 0 & 0 & 0 & l_{96} & 0 & l_{98} & 0\end{array}\right]$

Thus, the total length of connected edges for panel $i$ is

$e_{i}=\sum_{j}^{j \in A_{i}} l_{i j} v_{i j}$.

Here, $j$ is the number of panels adjacent to panel $i, j \in A_{i}$. In Fig. 6, since each panel is in unit size $l_{i j}=1$ and $v_{i j}=1$ (see the structural adjacent matrix Eq. (4)), the length of connected edges for panel 3 is $e_{3}=2$; similarly, $e_{6}=3$ and $e_{5}=4$. The total connected edges of a shared layer $k$ is determined by the panels it covers. Thus, the total length of connected edges of the shared layer $k$ is calculated through the layer adjacent matrix $\boldsymbol{V}^{l}$ :

$E_{k}=\sum_{i}^{i \in \bar{u}_{k}} \sum_{j}^{j \in A_{i}} l_{i j} v_{i j k}$,

where $j$ is the number of panels adjacent to panel $i$ and $i$ is the number of panels covered by the shared layer $k$. For instance, in Fig. 3(a), a global shared layer covers all panels: $E=24$; in Fig. 3(b), a subregion shared layer covers 6 panels: $E=12$; in Fig. 3(c), an individual layer covers only one panel, all the elements $v_{i j k}$ in $\boldsymbol{V}^{l}$ are 0 : $E=0$. Instinctually, each connected edge corresponds to a ply drop and it characterizes the blending property of the structure as well as the shape of shared layer. The number of connected edges is defined for an individual panel, but not between two adjacent panels, because connected edges/ply drops vary within a connected layer and hence are independent variables. To explain the definition, Fig. 7 gives a simple

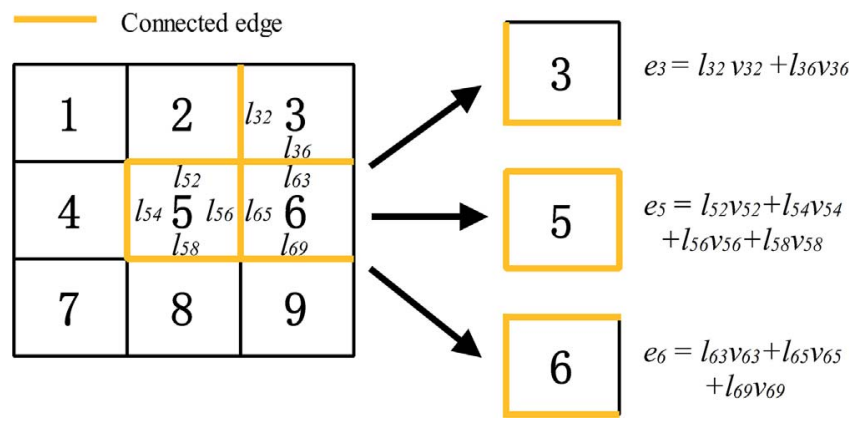

Fig. 6. Definition of connected edges. 


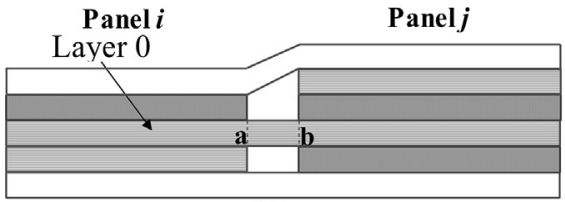

(a)

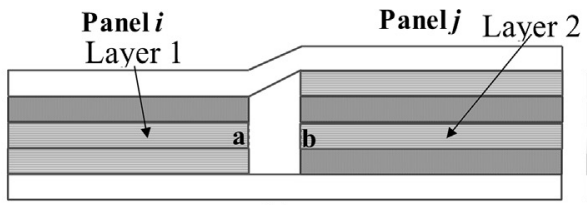

(b)

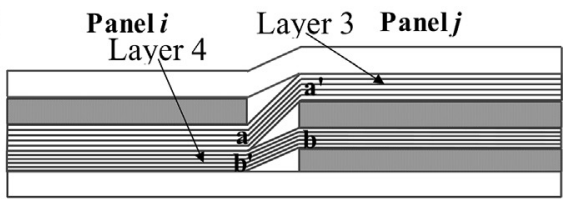

(c)

Fig. 7. Illustration of definition of connected edges: (a) original layer 0; (b) drop layers 1 and 2; (c) new shared layers 3 and 4.

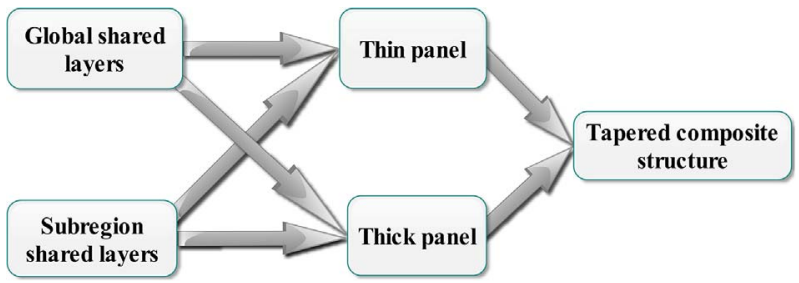

Fig. 8. Relationship between layers, panels and tapered composite structure.

example. Here, a blended layer 0 connects two panels $i$ and $j$ (see Fig. 7(a)): if there is a ply drop between panels $i$ and $j$ (see Fig. 7(b)), the connected edges are divided into two parts a and $\mathrm{b}$, and belonging to two layers 1 and 2 respectively. Subsequently, if they can be blended with other layers at different stacking positions of its adjacent panel (see Fig. 7(c)), such as connected edges a-a' and b-b', then two new shared layers 3 and 4 are formed, leading to a new structure.

\subsubsection{Panel continuity ratio}

In a tapered composite structure, the layer boundaries are controlled by thickness distribution, stacking sequence and manufacturing constraints. Since the layer shape can be evaluated according to the structural thickness distribution via the GSLB method [21], the maximum blending property is directly related to the structural thickness distribution. Fig. 8 demonstrates the relationship of layers, panels and a tapered composite structure. Considering a tapered composite structure composed of individual panels with different thicknesses, each panel is consisting of layers with various shape that cover multiple panels. The relationship between the shape of layers and thicknesses of panels should be involved to characterize the overall blending property of the structure.

As aforementioned, a new global blending model for an individual panel and the overall structure is developed, where thickness effects, information of connected edges (ply drops) and layer shapes are included. The panel continuity ratio is defined as

$C_{i}=\sum_{\theta=\theta_{1}}^{\theta_{M}} \sum_{k} \frac{n_{i k}^{\ominus}}{n_{i}} \cdot \frac{E_{k}}{E_{g}} \cdot \frac{S_{k}}{S_{g}}$,

where $i(i=1,2, \ldots, P)$ is the number of panel. $n_{i}$ is the total ply number of panel $i$ (half of the symmetric laminate). $n_{i k}^{\ominus}$ is the ply number of the shared layer $k$ with ply orientation $\theta$ in panel $i$ (when $k$ is varying from 1 to $Q, n_{i k}^{\theta}=1$ for each $k$ ), $\theta$ is the ply orientation and $k$ is the number of shared layers. $E_{k}$ is the total length of connected edges of the shared layer $k$ and $E_{g}$ is the total length of connected edges of a global shared layer. $S_{k}$ is the area of shared layer $k$ and $S_{g}$ is the area of a global shared layer.

\subsubsection{Ply-panel continuity ratio}

As the overall blending parameter: the ply-panel continuity ratio $C$ is defined as the average of panel continuity ratios

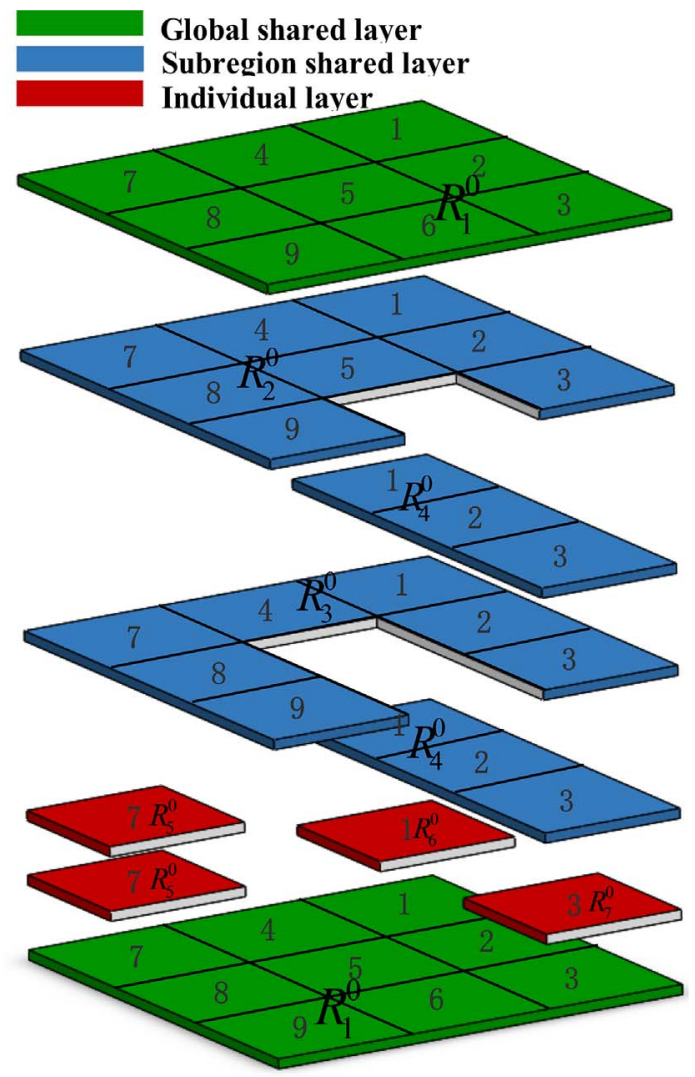

Fig. 9. Information of shared layers for the 9-panel structure.

$$
\begin{aligned}
& R_{1}^{0}: E_{1}=24, S_{1}=9, n_{i 1}^{0}=2, \\
& R_{2}^{0}: E_{2}=18, S_{2}=8, n_{i 2}^{0}=1, \\
& R_{3}^{0}: E_{3}=12, S_{3}=7, n_{i 3}^{0}=1, \\
& R_{4}^{0}: E_{4}=4, S_{4}=3, \quad n_{i 4}^{0}=2, \\
& R_{5}^{0}: E_{5}=0, S_{5}=1, \quad n_{i 5}^{0}=2, \\
& R_{6}^{0}: E_{6}=0, S_{6}=1, n_{i 6}^{0}=1, \\
& R_{7}^{0}: E_{7}=0, S_{7}=1, n_{i 7}^{0}=1,
\end{aligned}
$$


$C=\frac{1}{P} \sum_{i=1}^{P} C_{i}$

Three factors employed in Eq. (30) are $n_{i k}^{\ominus} / n_{i}, E_{k} / E_{g}$ and $S_{k} / S_{g} . n_{i k}^{\ominus} / n_{i}$ characterizes the thickness distribution, $E_{k} / E_{g}$ provides continuity/ boundary information and $S_{k} / S_{g}$ describes the size effect of shared layers. For instance, the three factors of a shared layer that covers 6 panels in Fig. 3(b) are $n_{i k}^{\ominus} / n_{i}=1 / n_{i}(i=2,4,5,6,8,9), E_{k} / E_{g}=12 / 24$ and $S_{k} / S_{g}=6 / 9$. When all the shared layers are stacked in the structure, the panel's continuity ratio can be evaluated. Algorithm 1 summarizes the evaluation process of the ply-panel continuity ratio.

Algorithm 1: Evaluation of ply-panel continuity ratio

(1) Input all shared layers' information $(k=1,2, \ldots, Q)$ : panels covered by shared layers $\overline{\boldsymbol{u}}_{s_{k}}$ (Eq. (13)), the shared-layer adjacent

matrix $V^{l}$ (Eq. (5)) and the connected edge matrix $L$ (Eq. (27));

(2) Calculate the area $S_{k}$ (Eq. (26)) and the connected edge $E_{k}$ (Eq. (29)) for each shared layer respectively, and save the connected edge $E_{g}$ and area $S_{g}$ of the global shared layer;

(3) Evaluate the panel continuity ratio $C_{i}(i=1,2, \ldots, P)$ from the first panel to the last one with multiple ply orientations (Eq. (30));

(4) Evaluate the ply-panel continuity ratio $C$ (Eq. (31)).

Note that the ply-panel continuity ratio is only related to the shapes of shared layers, but independent of their stacking sequences.

\subsection{A simple example}

The 9-panel structure with only $0^{\circ}$ layers in Fig. 9 is adopted to demonstrate the evaluation of the ply-panel continuity ratio. Since some shared layers are with the same shape, we use $R_{k}^{\theta}$ to denote the shape of shared layer $k, \theta$ is the ply orientation. Here the subscript $k$ represents the number of different shape shared layers, thus the parameter $n_{i k}^{\ominus}$ is not equal to 1 in Eq. (30). For instance in Fig. 9, there are 10 shared layers with only 7 different shapes. The ply number of same shape shared layer $R_{k}^{\ominus}$ is $n_{i k}^{\ominus}$. The length of connected edges $E_{k}$, areas $S_{k}$ and the number of $n_{i k}^{\ominus}$ are summarized in Table 1 . The panel continuity ratio and ply-panel continuity ratio can be evaluated by Algorithm 1, the results are given in Table 1.

A shared layer often covers more than one panel and layers in a panel at different stacking positions belong to shared layers with different shapes. Thus, the panel continuity ratio $C_{i}$ is controlled by the number of shared layers and their shapes in the panel $i$. Fig. 10(a) gives the relationship between the panel continuity ratio and shared layers. According to Table 1, if a panel consists of shared layers with big areas, its panel continuity ratio is big (e.g. panels $4,5,6,8$, 9); otherwise, the panel continuity ratio is small (e.g. panels $1,2,3,7$ ). Consequently, a thin panel (e.g. panels 5 and 6) consists of global shared layers or shared layers with big areas has a good continuity property, which makes a higher contribution to the overall blending property. On the contrary, a thick panel (e.g. panels 1 and 3) with many ply drops has a poor continuity property, making a lower contribution to the overall blending property (see Fig. 10(b)). As shown in Table 1, the thinnest panel 6 has the maximum panel continuity ratio (equal to 1 ), while the thickest panel 1 has the minimum panel continuity ratio.

According to Eq. (30) and Fig. 10, if all shared layers are extended to global shared layers, there is no ply drop in every shared layer and each shared layer is with maximum area. Furthermore, it can be derived from Eqs. (29) to (31) that a structure only consists of global shared layers has the maximum ply-panel continuity ratio, which requires all panels having the same thickness (uniform thickness structure), with each panel's continuity ratio being equal to 1 . That implies maximizing the ply-panel continuity ratio is equivalent to minimize the total number of ply drops in the structure. The global blending model is related to ply drops of each panel and hence it is effective to characterize the global blending property of the tapered composite structures.

The above discussions indicate that the global shared layer has a maximum contribution to the blending property of the entire structure. On the contrary, an individual layer has no connected edges, but increases thickness, thus, individual layers should be avoided if possible (see Fig. 10).

\subsection{How to use the global blending model?}

The above example illustrates how the proposed global blending model characterizes the blending property of a tapered composite structure. Another important application of the proposed global blending model is that it can guide the maximum blending design when thicknesses of the structure are varying. When layers are added to (or deleted from) the structure, there are several problems to address. First, which panel the layers should be added to? It is determined by the stiffness of each panel, insufficient stiffness leads to an add-layer operation. After that, the other problems are:

(1) What kind of ply orientation should be chosen for add-layer operation?

(2) Which stacking position is the best choice?

(3) Which shared layer the added layer should be blended with?

Table 1

Information of shared layers for evaluation of ply-panel continuity ratio.

\begin{tabular}{|c|c|c|c|c|c|c|c|c|c|c|}
\hline \multirow{5}{*}{$\begin{array}{l}\text { Panel number } \\
\quad i\end{array}$} & \multirow[t]{5}{*}{ Thickness distribution $n_{i}$} & \multicolumn{8}{|c|}{ Connected edges $E_{j}$ and area $S_{j}$ of shared layers } & \multirow[t]{5}{*}{ Panel continuity ratio $C_{i}$} \\
\hline & & & $R_{1}^{0}$ & $R_{2}^{0}$ & $R_{3}^{0}$ & $R_{4}^{0}$ & $R_{5}^{0}$ & $R_{6}^{0}$ & $R_{7}^{0}$ & \\
\hline & & $k$ & 1 & 2 & 3 & 4 & 5 & 6 & 7 & \\
\hline & & $E_{k}$ & 24 & 18 & 12 & 4 & 0 & 0 & 0 & \\
\hline & & $S_{k}$ & 9 & 8 & 7 & 3 & 1 & 1 & 1 & \\
\hline 1 & 7 & \multirow{9}{*}{$\begin{array}{l}\text { Ply number } \\
n_{i k}^{\ominus} \text { of } \\
\text { shared layer } R_{k}^{\ominus}\end{array}$} & 2 & 1 & 1 & 2 & - & 1 & - & 0.4524 \\
\hline 2 & 6 & & 2 & 1 & 1 & 2 & - & - & - & 0.5278 \\
\hline 3 & 7 & & 2 & 1 & 1 & 2 & - & - & 1 & 0.4524 \\
\hline 4 & 4 & & 2 & 1 & 1 & - & - & - & - & 0.7639 \\
\hline 5 & 3 & & 2 & 1 & - & - & - & - & - & 0.8889 \\
\hline 6 & 2 & & 2 & - & - & - & - & - & - & 1.0000 \\
\hline 7 & 6 & & 2 & 1 & 1 & - & 2 & - & - & 0.5093 \\
\hline 8 & 4 & & 2 & 1 & 1 & - & - & - & - & 0.7639 \\
\hline 9 & 4 & & 2 & 1 & 1 & - & - & - & - & 0.7639 \\
\hline \multicolumn{3}{|c|}{ Ply-panel continuity ratio $C$} & & & & & & & & 0.6803 \\
\hline
\end{tabular}

Notations: for example, using Eq. (30): $C_{1}=\frac{2}{7} \cdot \frac{24}{24} \cdot \frac{9}{9}+\frac{1}{7} \cdot \frac{18}{24} \cdot \frac{8}{9}+\frac{1}{7} \cdot \frac{12}{24} \cdot \frac{7}{9}+\frac{2}{7} \cdot \frac{4}{24} \cdot \frac{3}{9}+\frac{1}{7} \cdot \frac{0}{24} \cdot \frac{1}{9}=0.4524$. 


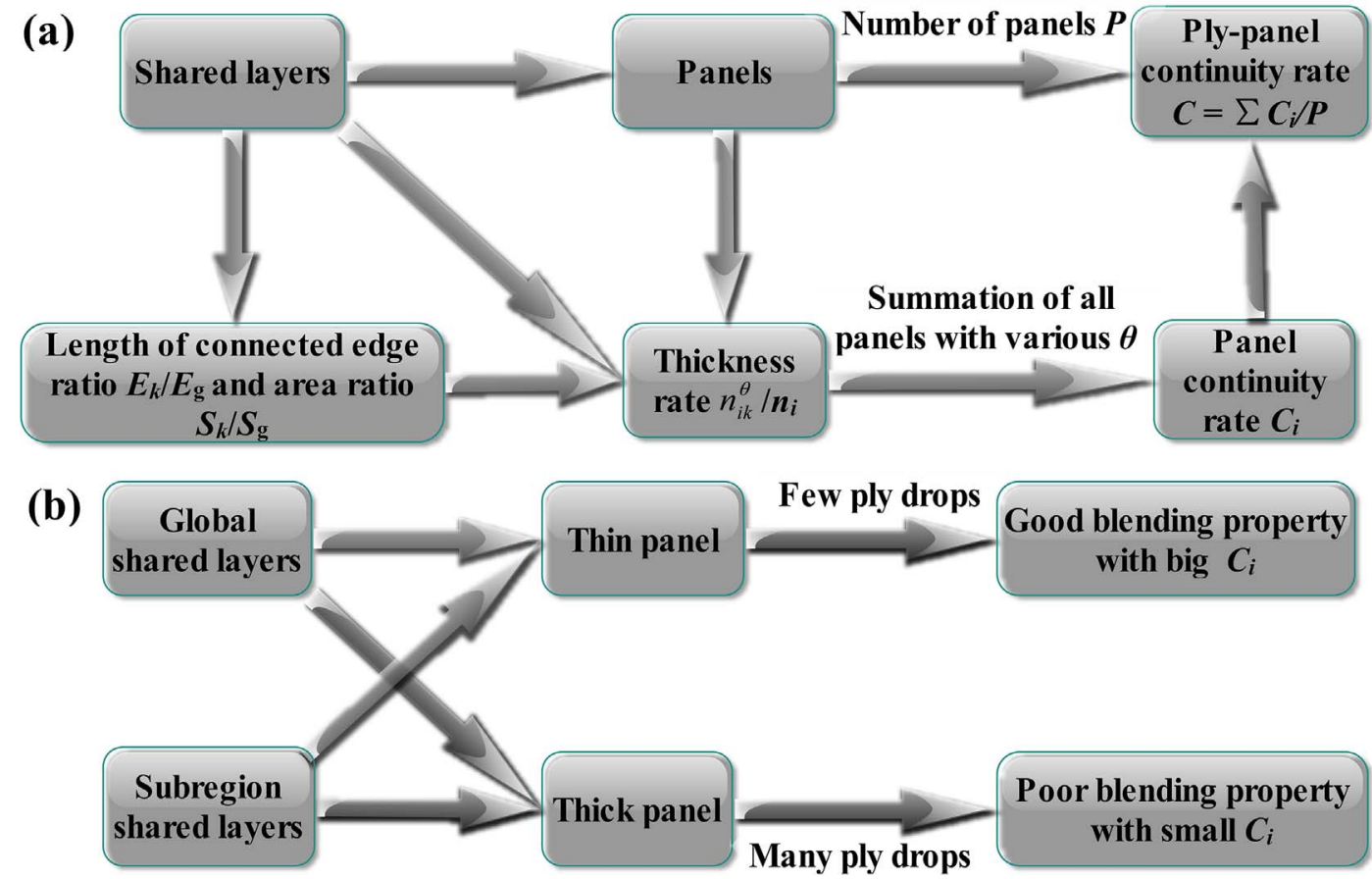

Fig. 10. Relationship of thickness, shared layers and continuity property: (a) evaluation process of ply-panel continuity ratio; (b) thickness effect on the continuity property.

The above three problems can be addressed by the proposed global blending model. From the researcher's perspective, the ply-panel continuity ratio is a "sensitive parameter" to the global blending property. That is to say each time a layer is added, if the stiffness of the panel is sufficient, the layer's best ply orientation, stacking position and the shared layer it blended with should maximize the ply-panel continuity ratio $C$ to improve the global blending property. However, the application of the global blending model requires another two design techniques: add-layer and exchange-blend operations, which will be introduced in the next section.

\section{Application of global blending model in blending design}

Since there are multiple choices of ply orientations between adjacent laminates in a tapered composite structure, the blending problem is complicated and tough to deal with. The proposed GBM can not only assess the global blending property, but also guide the blending design process. The application of the GBM in blending design are combined with add-layer and exchange-blend operations:

(1) It can identify the exact stacking position when add (delete) a layer.

(2) It can determine which ply orientation should be exchanged, and

\begin{tabular}{|c|c|c|c|c|c|c|c|c|}
\hline \multicolumn{9}{|c|}{ Panel number } \\
\hline 1 & 2 & 3 & 4 & 5 & 6 & 7 & 8 & 9 \\
\hline 45 & 45 & 45 & 45 & 45 & $\overline{45}$ & 45 & 45 & $\overline{45}$ \\
\hline 0 & & & & & & & & \\
\hline-45 & -45 & -45 & -45 & -45 & -45 & -45 & -45 & -45 \\
\hline & & & $\mathbf{0}$ & & & $\mathbf{0}$ & $\mathbf{0}$ & $\mathbf{0}$ \\
\hline 0 & 0 & 0 & 0 & 0 & 0 & 0 & 0 & 0 \\
\hline 45 & 45 & 45 & 45 & 45 & 45 & 45 & 45 & 45 \\
\hline 90 & 90 & 90 & 90 & 90 & 90 & 90 & 90 & 90 \\
\hline-45 & -45 & -45 & -45 & -45 & -45 & -45 & -45 & -45 \\
\hline
\end{tabular}

(a) which shared layer should be blended with.

In these two operations, the first concern is about a kind of ply orientations chosen for adding/exchanging, the second concern is about a shared layer that identified for the new added/exchanged layer to blend with. The stacking position of the new added/exchanged layer is determined by the original shared layer it blend with. Thanks to the GBM, these two operations are coupled together to find the maximum blending design. Additionally, to obtain the maximum blending property, the manufacturing constraints CT3 to CT4 are released in addlayer and exchange-blend operations, and they are taken into account together with CT5 and CT6 in a sequence-adjustment operation. In what follows, the GBM based add-layer, exchange-blend and sequenceadjustment operations are introduced.

\subsection{Add-layer operation}

Sometimes, layers are added to increase stiffness of individual panels to satisfy buckling constraint, or to adjust the proportion of ply orientations to satisfy ply orientation mismatch constraint CT7. Here, the ply orientations of added layers are limited to $0^{\circ}$ or $90^{\circ}$, thus the balance requirement is released for the add-layer operation and,

\begin{tabular}{|c|c|c|c|c|c|c|c|c|}
\hline \multicolumn{9}{|c|}{ Panel number } \\
\hline 1 & 2 & 3 & 4 & 5 & 6 & 7 & 8 & 9 \\
\hline 45 & 45 & 45 & $\overline{45}$ & 45 & 45 & 45 & 45 & 45 \\
\hline-45 & -45 & -45 & -45 & -45 & -45 & -45 & -45 & -45 \\
\hline 0 & & & 0 & & & 0 & 0 & 0 \\
\hline 0 & $\mathbf{0}$ & $\mathbf{0}$ & $\mathbf{0}$ & $\mathbf{0}$ & $\mathbf{0}$ & $\mathbf{0}$ & $\mathbf{0}$ & $\mathbf{0}$ \\
\hline 45 & 45 & 45 & 45 & 45 & 45 & 45 & 45 & 45 \\
\hline 90 & 90 & 90 & 90 & 90 & 90 & 90 & 90 & 90 \\
\hline-45 & -45 & -45 & -45 & -45 & -45 & -45 & -45 & -45 \\
\hline
\end{tabular}

(b)

Fig. 11. Add-layer operation: (a) Case 1; (b) Case 2. 
therefore, there are only two cases for adding a layer:

Case 1: Add as an individual layer $\theta$.

Case 2: Add to a stacking position and blend to the original layer.

The ply orientation of the add layer is denoted by $\theta, \theta=0^{\circ}$ or $90^{\circ}$. Fig. 11 depicts the two cases, in which value in red is the added layer, the row in grey is the new layer and the empty stacking positions are ply drops. To maximize the global blending property, in Case 2, if multiple shared layers are available for blending, the one with maximum ply-panel continuity ratio $C$ is selected for the add-layer operation. The add-layer algorithm is summarized in Algorithm 2. Note that ply-panel continuity ratio $C$ is only related to the shape of shared layers in the structure. Hence, in case 1 the individual layer is always inserted at outermost stacking position without violation of constraints, while in case 2 the added layer is blended with a shared layer at its original stacking position.

\section{Algorithm 2: Add-layer operation}

(1) For the panel $i$ (panel $i$ is with insufficient stiffness), choose a ply orientation $\theta$ from $0^{\circ}$ or $90^{\circ}$.

(2) Case 1: Insert the layer as an individual layer and evaluate the ply-panel continuity ratio $C$ and save it, also save the corresponding $\boldsymbol{\Omega}$ and reset it. Find the adjacent panels $j$ of panel $i$ according to Eq. (4).

(3) Case 2: Identify a shared layer with ply orientation $\theta$ that covers the adjacent panel $j$ but drops at panel $i$, add the layer and blend with the shared layer. Evaluate the ply-panel continuity ratio $C$ and save it. Save the corresponding $\boldsymbol{\Omega}$ and reset it.

(4) If more than one shared layers are available for blending in (3), repeat (3) for all possible shared layers. Choose and save the one with maximum ply-panel continuity ratio $C$ with its corresponding stacking sequence matrix $\boldsymbol{\Omega}$.

(5) Compare the ply-panel continuity ratios for Case 1 and 2, select and save the one with the maximum ply-panel continuity ratio $C$ with its corresponding stacking sequence matrix $\boldsymbol{\Omega}$.

(6) Repeat the above procedures for those panels whose stiffnesses are insufficient.

\subsection{Exchange-blend operation}

Since only $0^{\circ}$ or $90^{\circ}$ layers are added in the add-layer operation, to improve the global blending property, some of them are exchanged and blended to bigger shared layers. There are 5 cases for different ply orientations to exchange to another one:
(1) $\pm \theta_{1} \rightarrow \pm \theta_{2}, \quad \theta_{1} \neq \theta_{2} ; \theta_{1}, \quad \theta_{2} \neq$

(2) $0_{2} \rightarrow \pm \theta$

(3) $90_{2} \rightarrow \pm \theta$

(4) $\pm \theta \rightarrow 90_{2}$

(5) $\pm \theta \rightarrow 0_{2}$

To demonstrate the exchange-blend operation, in Fig. 12: consider the exchange-blend operation between two adjacent panels 3 and 4 (two blue columns), if two $90^{\circ}$ layers (values in red in Fig. 12(a)) in panel 4 are exchanged to another ply orientation to improve the blending property, there are multiple choices since in panel 3 there are four candidate ply orientations $\pm 30^{\circ}, \pm 45^{\circ}, \pm 60^{\circ}, \pm 75^{\circ}$ to blend with. However, some of them are originally global shared layers $\left( \pm 45^{\circ}, \pm 60^{\circ}\right.$ ), only $\pm 75^{\circ} / \pm 30^{\circ}$ are available for exchange-blend operation. In panel 4 , two $90^{\circ}$ layers (values in red in Fig. 12(a)) are exchanged to a pair of $\pm 75^{\circ} / \pm 30^{\circ}$ layers (in orange background in Fig. 12(b) and (c), respectively). Still, it is necessary to assess which one is better for improving the blending property. The ply-panel continuity ratio is calculated to make the decision. Obviously, case 2 (2) in Fig. 12(c)) is the better one since the area of the new shared layer (rows in grey) is bigger and, hence, has a bigger ply-panel continuity ratio. For the whole structure, between every two adjacent panels, all ply orientations in one panel should be detected for exchange-blend operation associated with the ply orientations in its adjacent panel. After each exchange-blended operation, the ply-panel continuity ratio is evaluated. The stacking sequence with maximum ply-panel continuity ratio is saved for further operation.

Detailed algorithm is summarized in Algorithm 3

\section{Algorithm 3: Exchange-blend operation}

for $i=1: P$

Find $\theta_{1}$ of panel $i$ and $\theta_{2}$ of its adjacent panel $j, \theta_{1} \neq \theta_{2}$.

for $k=1: Q$

In panel $i$, find the row numbers of $\pm \theta_{1}$ and $\pm \theta_{2}$, respectively. Then delete the

original $\pm \theta_{1}$ and exchange to $\pm \theta_{2}$, finally blend to the new shared layers with $\pm \theta_{2}$ at

the stacking positions of $\pm \theta_{2}$. After blending, calculate the buckling load factor

$\lambda_{c b}\left(\zeta_{i}\right)$ and the ply-panel continuity ratio $C$.

if $\lambda_{c b}\left(\zeta_{i}\right)>1$

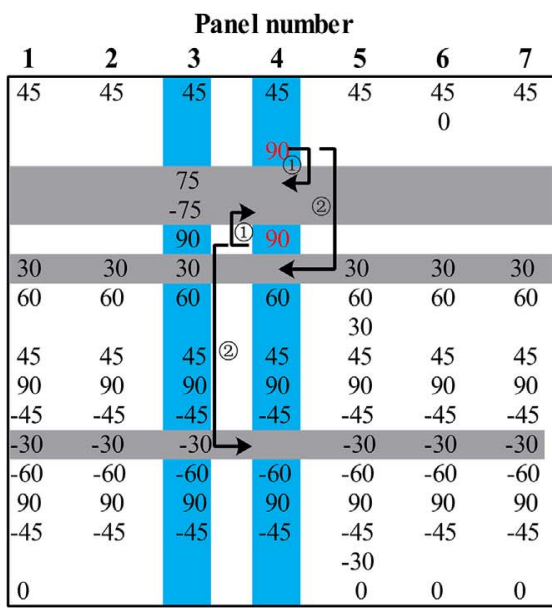

(a)

\begin{tabular}{|c|c|c|c|c|c|c|}
\hline \multicolumn{7}{|c|}{ Panel number } \\
\hline 1 & 2 & 3 & 4 & 5 & 6 & 7 \\
\hline \multirow[t]{3}{*}{45} & 45 & 45 & 45 & 45 & 45 & 45 \\
\hline & & $\begin{array}{r}75 \\
-75\end{array}$ & $\begin{array}{r}75 \\
-75\end{array}$ & & & \\
\hline & & 90 & & & & \\
\hline 30 & 30 & 30 & & 30 & 30 & 30 \\
\hline 60 & 60 & 60 & 60 & 60 & 60 & 60 \\
\hline & & & & 30 & & \\
\hline 45 & 45 & 45 & 45 & 45 & 45 & 45 \\
\hline 90 & 90 & 90 & 90 & 90 & 90 & 90 \\
\hline-45 & -45 & -45 & -45 & -45 & -45 & -45 \\
\hline-30 & -30 & -30 & & -30 & -30 & -30 \\
\hline-60 & -60 & -60 & -60 & -60 & -60 & -60 \\
\hline 90 & 90 & 90 & 90 & 90 & 90 & 90 \\
\hline-45 & -45 & -45 & -45 & -45 & -45 & -45 \\
\hline & & & & $\begin{array}{c}-30 \\
0 \\
\end{array}$ & 0 & 0 \\
\hline
\end{tabular}

(b)

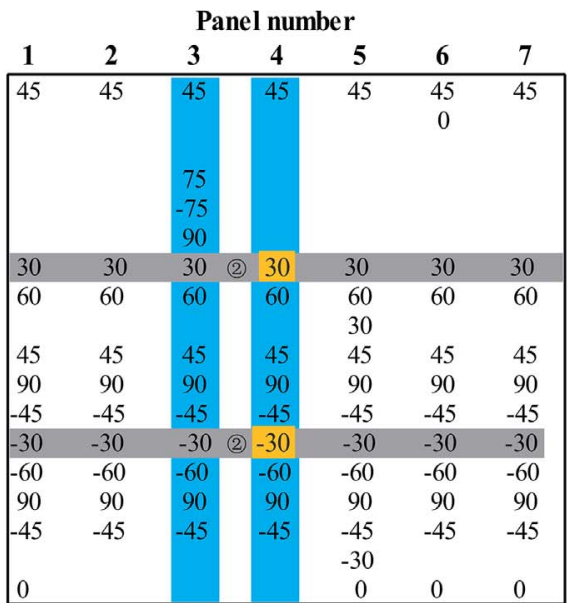

(c)

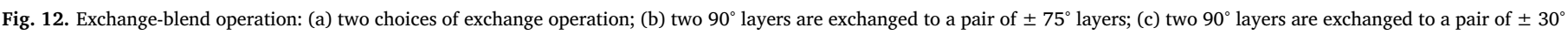
layers. 
Algorithm 3: Exchange-blend operation

Save the ply-panel continuity ratio $C$ and the stacking sequence matrix $\boldsymbol{\Omega}$.

else

Reset the $\boldsymbol{\Omega}$.

end if

end for

Choose the maximum ply-panel continuity ratio $C$ with its corresponding stacking

sequence matrix $\boldsymbol{\Omega}$.

end for

\subsection{Sequence-adjustment operation}

Note that in the above two operations, the manufacturing constraints CT3 and CT4 were released to obtain the maximum blending property. In this section, the manufacturing constraints CT3 to CT6 are involved in the sequence-adjustment operation.

However, these constraints are difficult to consider simultaneously, to simplify the design procedure, the sequence-adjustment operation first checks the stacking position only violates CT3 or CT4 in $\boldsymbol{\Omega}$. If a layer in an individual panel violates the constraints CT3 or CT4, the layer based shared layer is adjusted to another stacking position, which is changing from the outermost to the mid-plane. The first stacking position that not violates the constraints CT3 or CT4 is saved for further operation.

The way to reduce the contiguity and simultaneous ply drops is more complicated when involves the previous two constraints CT3 and CT4. For one shared layer, the total number of contiguity ply drops is related to the up and down shared layers that cover all the ply drops of the current shared layer, thus the constraints CT5 can only be satisfied gradually, which requires adjustment of multiple shared layers. For instance, in Fig. 13, the constraints are checked sequentially: assume that the shared layer $\omega_{r_{3}}^{3}$ (the shared layer with yellow arrow in Fig. 13(a)) is adjusted, first obtain the total number of contiguity ply drops (Eq. (A.4)) of the shared layer $\omega_{r_{3}}^{3}$ at its stacking position. Since it covers panels 2 and 3, the total number of contiguity ply drops is the sum of the number of contiguity ply drops of panels 2 and 3 , which is related to the up and down shared layers of the current shared layer $\omega_{r_{3}}^{3}$ (see Fig. 13(a)): in panel 2 the number of contiguity ply drops is 5 (sum of the black arrows on two edges of panel 2), while in panel 3 the number of contiguity ply drops is 6 (sum of the black arrows on one edge of panel 3). Thus, the total number of contiguity ply drops of the shared layer $\omega_{r_{3}}^{3}$ is 11 . Then delete the shared layer $\omega_{r_{3}}^{3}$, the constraints CT3 and CT4 for individual panels' stacking sequences $\zeta_{2}$ and $\zeta_{3}$ should be checked for panels 2 and 3 respectively. If the constraints CT3 and CT4 are satisfied, insert the shared layer $\omega_{r_{3}}^{3}$ to another stacking position (see Fig. 13(b)), and recheck the constraints CT3 to CT4 for $\zeta_{2}$ and $\zeta_{3}$ respectively. If constraints CT3 and CT4 are satisfied, evaluate the buckling load factors of panels 2 and 3 and ensure they are bigger than one. Simultaneously, after the shared layer $\omega_{r_{3}}^{3}$ is adjusted, count the total number of contiguity ply drops at the current stacking position and make sure that the number of contiguity ply drops (or the simultaneous ply drops) at the current stacking position is decreased after adjustment. As can be seen in Fig. 13(b), for the shared layer $\omega_{r_{3}}^{3}$ at the current position, the total number of contiguity ply drops is 6 (since laminates are symmetrical about the mid-plane), which is smaller than 11 when $\omega_{r_{3}}^{3}$ is at its original stacking position (Fig. 13(a)). However, in panel 3, number of contiguity ply drops is 5 after adjustment (Fig. 13(b)), which violates constraint CT5. That implies multiple shared layers should be adjusted to satisfy constraint CT5. For all subregion shared layers, the above sequence adjustment procedures are performed, each time a subregion shared layer is deleted at its original stacking position and inserted from the outermost to the mid-plane. The best inserted stacking position with a minimum total number of contiguity ply drops is chosen, and its corresponding stacking sequence is saved for further operation. Within the adjustment of subregion shared

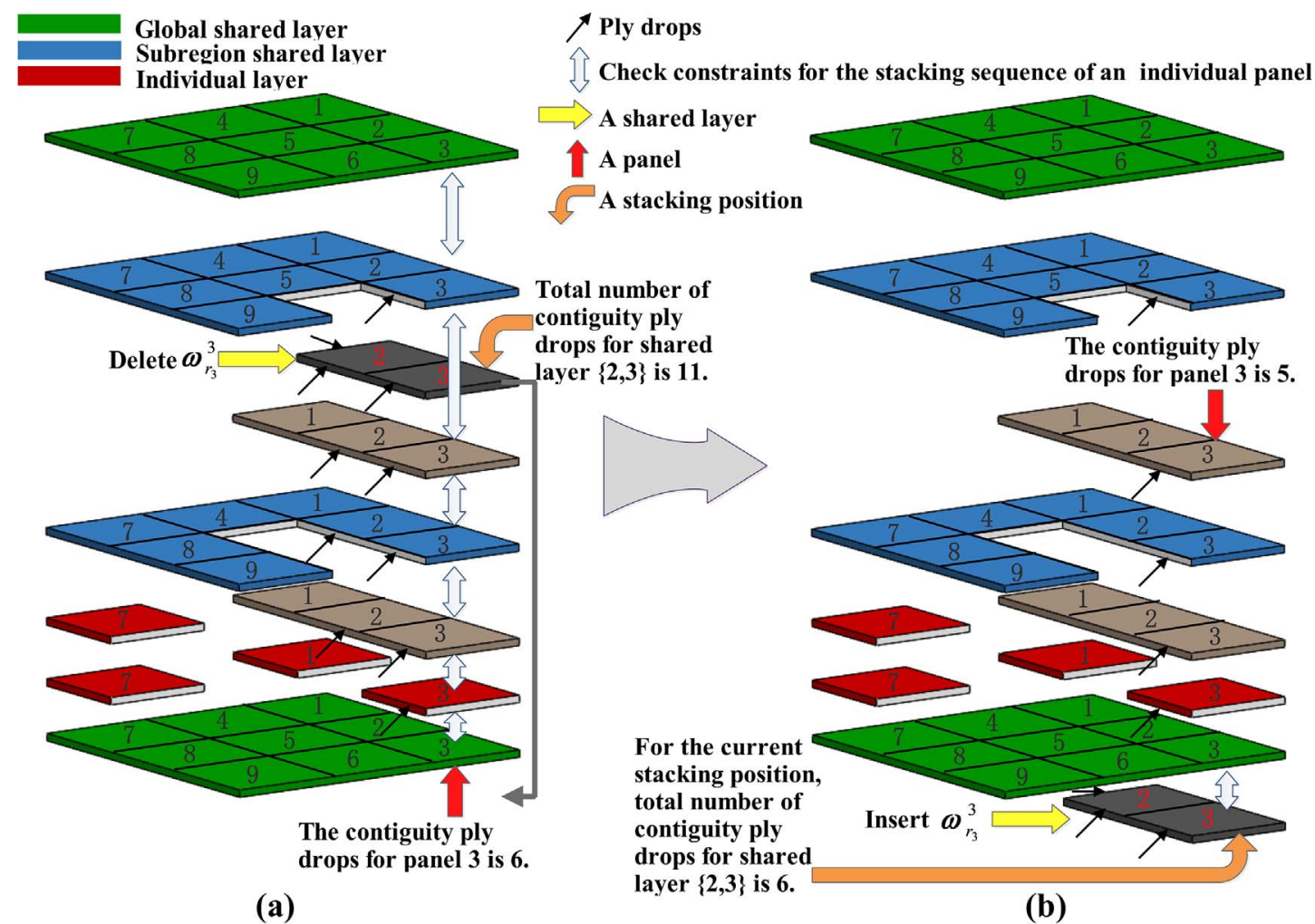

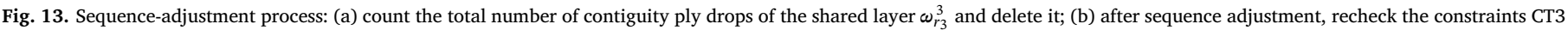
and CT4 and ensure the total number of contiguity ply drops is decreased at the current stacking position compare to that of its original stacking position. 


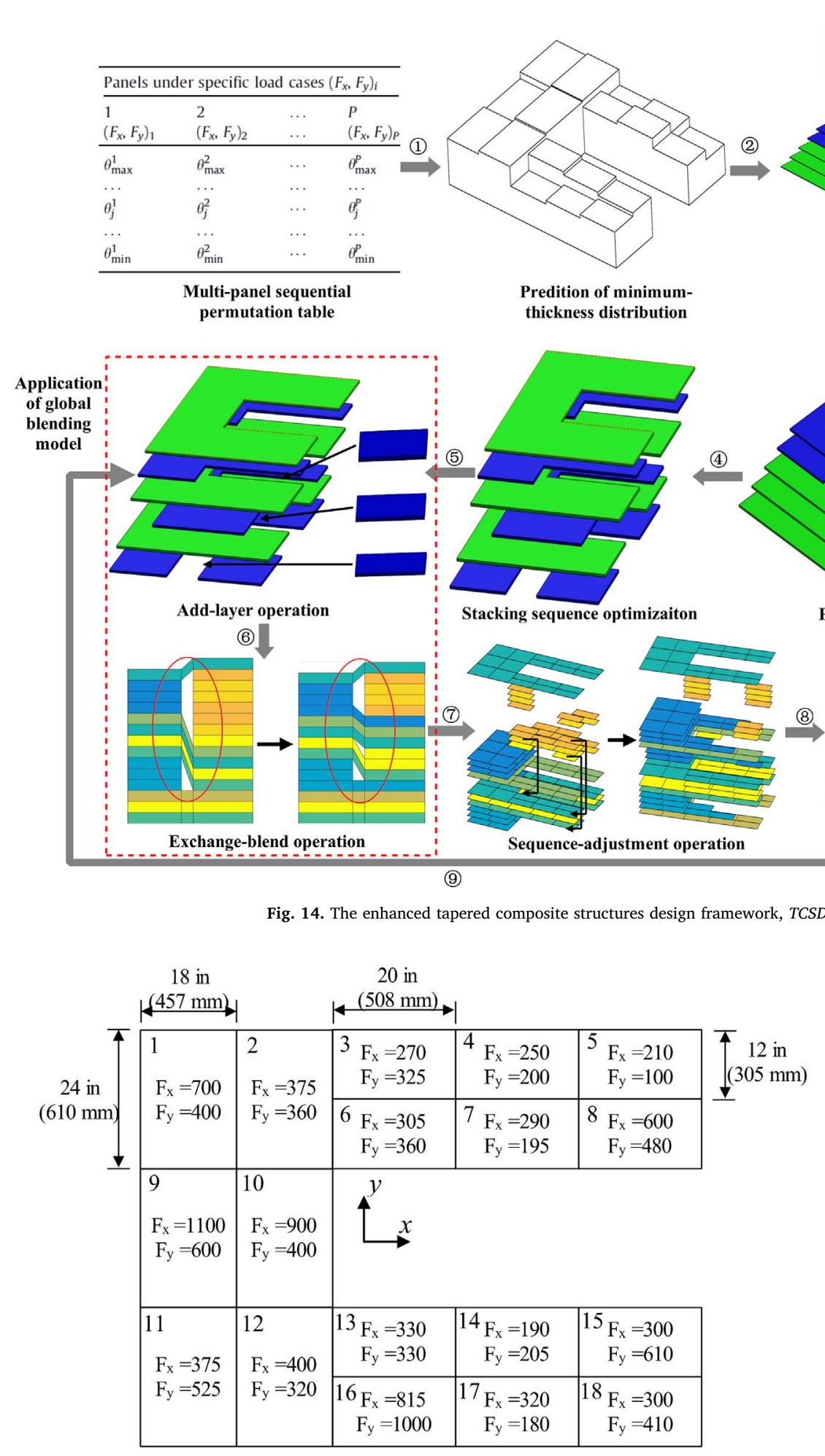

(a)

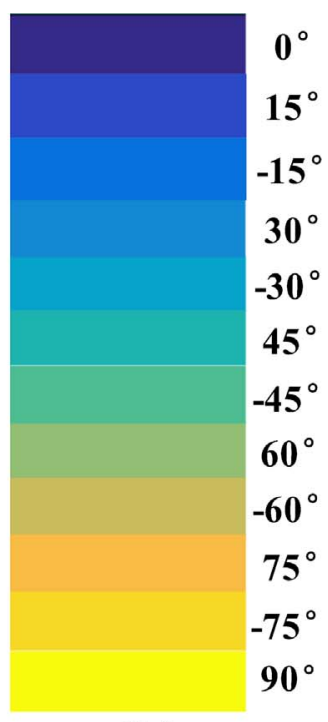

(b)

Fig. 15. 18-panel problem: (a) geometry, loads (all loads in lbf/in; $1 \mathrm{lbf} / \mathrm{in}=175.127 \mathrm{~N} / \mathrm{m}$ ) and sections along $x$ and $y$ axial; (b) legend of ply orientations.

layers, the constraints CT5 and CT6 are satisfied gradually. Note that, the sequence-adjustment operation is only performed for the subregion shared layers, but not for the global shared layers. The detailed algorithm is given in Algorithm 4: if vio_CTi is equal to 1, the constraint CTi is violated in the sequence-adjustment operation.
Algorithm 4: Sequence-adjustment operation

$k=1$; Success_sign $1=0$; Success_sign $2=0$;

while $k \leq Q$

for $i=1: P$ 


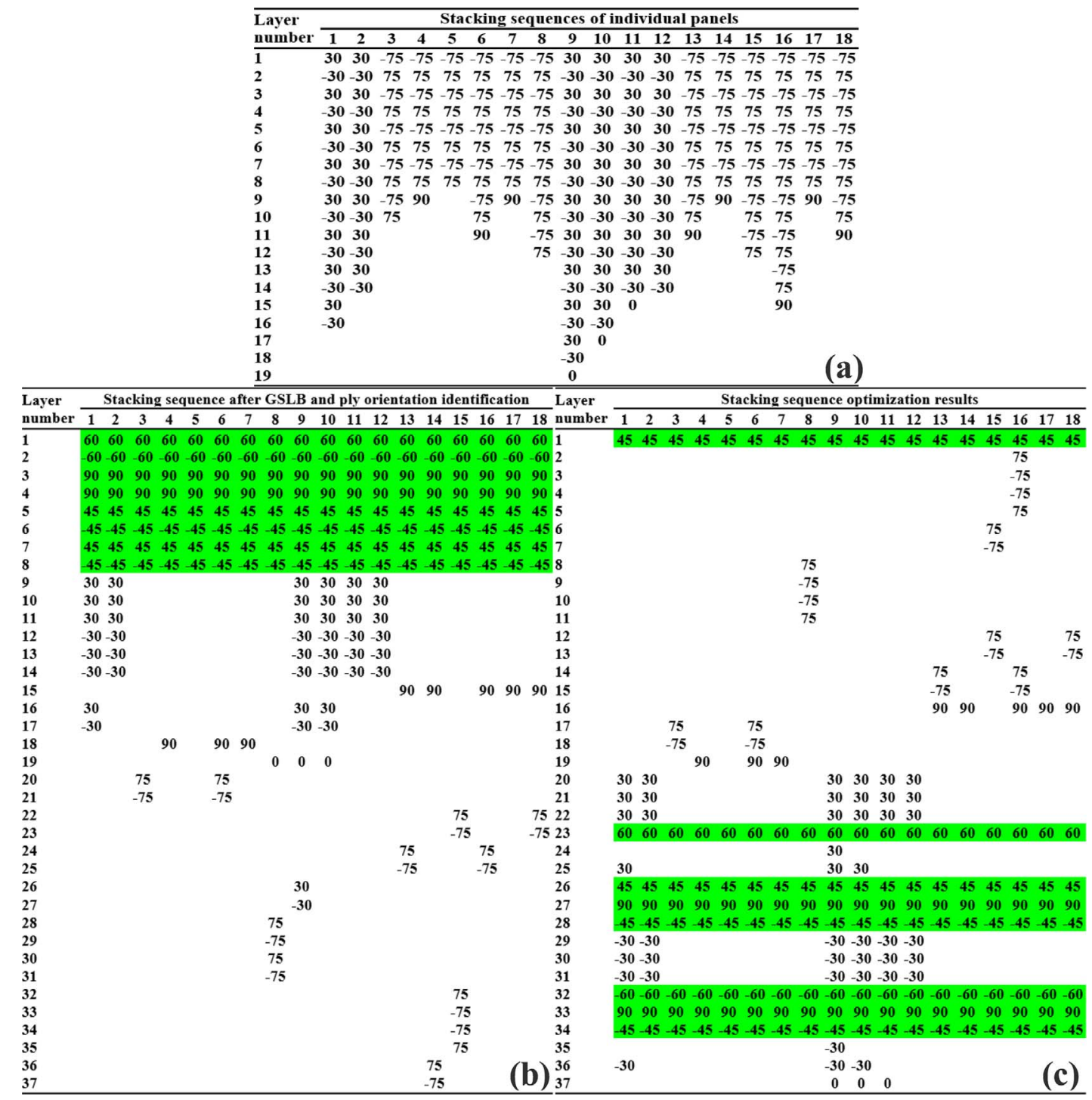

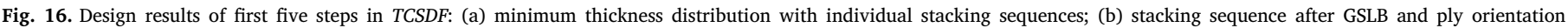
identification; (c) stacking-sequence optimization results.

\section{Algorithm 4: Sequence-adjustment operation}

Check the constraints CT3 and CT4 for the $\zeta_{i}$ and $\boldsymbol{\Omega}_{k i}$, respectively.

$$
\begin{aligned}
& \text { if vio_CT3= }=1 \| \text { vio_CT4 }==1 \\
& \quad \text { for } r=k+1: Q \| r=2:-1: k-1
\end{aligned}
$$

Insert the shared layer $\omega_{r_{k}}^{k}$ to the stacking position $r$.

\section{Check the}

constraints CT3 and CT4 for the $\zeta_{i}$ and $\boldsymbol{\Omega}_{r i}$, respectively, and calculate

$$
\begin{aligned}
& \lambda_{c b}\left(\zeta_{i}\right) . \\
& \text { if } v i o_{-} \mathrm{CT} 3==0 \& \& v i o_{-} \mathrm{CT} 4==0 \& \& \lambda_{c b}\left(\zeta_{i}\right)>1
\end{aligned}
$$

Save the stacking sequence matrix $\boldsymbol{\Omega}$ and the row number $r$ in an array

\section{ban_num.}

Success_sign $1=1$;

break;

end if

end for

if Success_sign $1==1$

$$
k=k+1 \text {; }
$$

break;

\section{Algorithm 4: Sequence-adjustment operation}

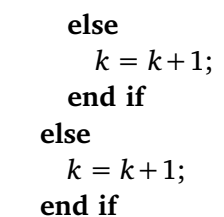

end for

end while

$k=1$; delete_sign $=0$;

while $k \leq Q$

Ensure $k$ is not in the array ban_num.

Obtain the total number of contiguity ply drops for the shared

layer $\omega_{r_{k}}^{k}$, then

delete the shared layer $\omega_{r_{k}}^{k}$ at stacking position $k$. Save the

stacking sequence

matrix $\boldsymbol{\Omega}$ and get $\overline{\boldsymbol{u}}_{s k}$.

for $i=1$ :length $\left(\overline{\boldsymbol{u}}_{s_{k}}\right)$

Check the constraints CT4 for the $\boldsymbol{\Omega}_{k i}$.

if $v$ io_CT $4 \neq 1$

delete_sign $=1$; 


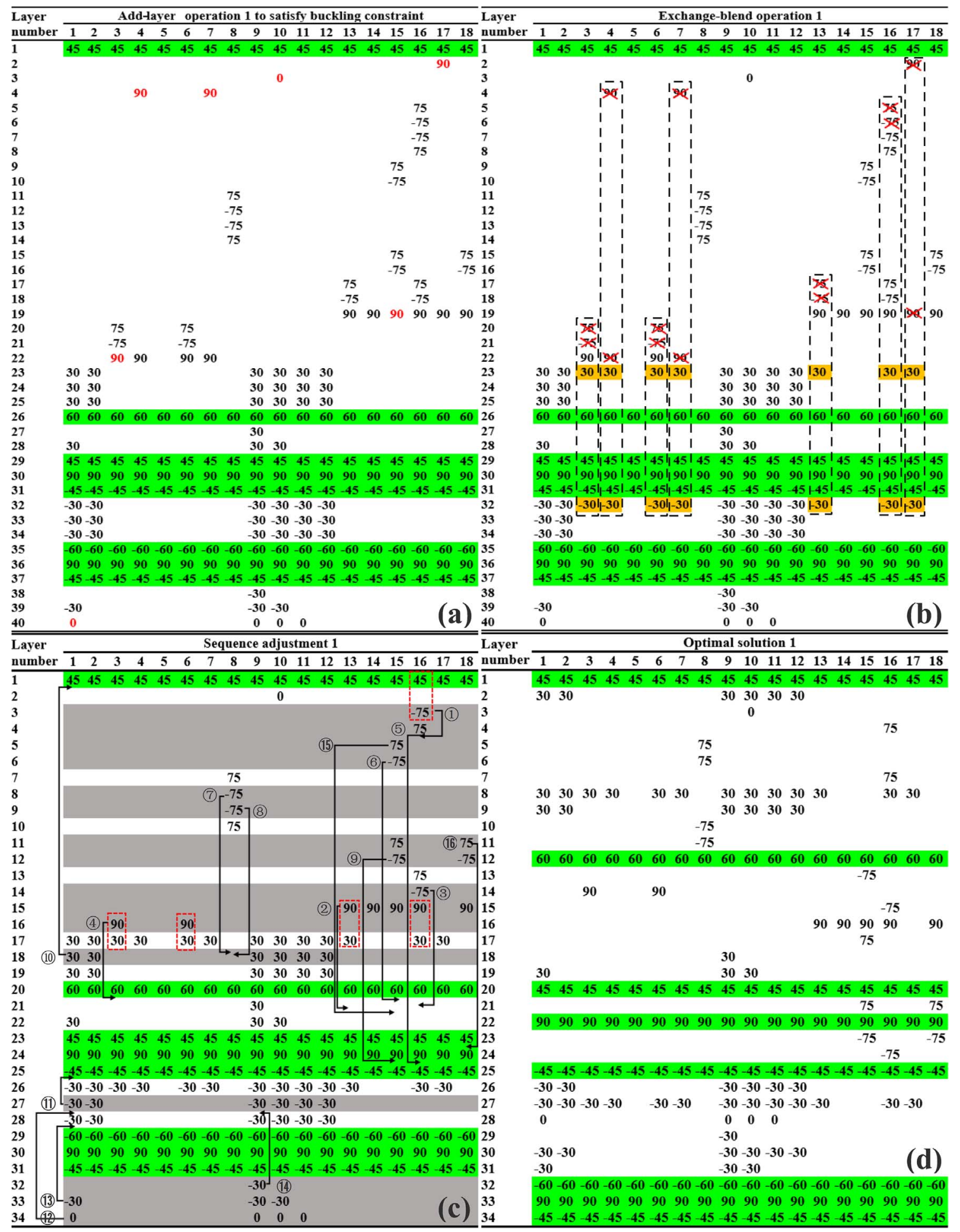

Fig. 17. First loop of (a) add-layer to improve stiffnesses of panels, (b) exchange-blend to improve global blending property and (c) sequence-adjustment to decrease contiguity ply drops; (d) Optimal solution 1 .

\section{Algorithm 4: Sequence-adjustment operation}

else

delete_sign $=0$;

$k=k+1$;

end if

end for

If delete_sign $==1$

for $r=k+1: Q \| r=2:-1: k-1$

\section{Algorithm 4: Sequence-adjustment operation}

Insert the shared layer $\omega_{r_{k}}^{k}$ to the stacking position $r$.

for $i=1$ :length $\left(\overline{\boldsymbol{u}}_{s_{k}}\right)$

Check the constraints CT3, CT4, CT5 and CT6 for the $\zeta_{i}$ and $\boldsymbol{\Omega}_{k i}$, and evaluate

$\lambda_{c b}\left(\zeta_{i}\right)$

if $\left(v i o_{-}\right.$CT $3 \neq 1 \& \& v i o_{-}$CT $\left.4 \neq 1\right) \& \&\left(v i o_{-}\right.$CT5 $\neq 1 \| v$ vio_CT6 $\left.\neq 1\right) \&$

$\& \lambda_{c b}\left(\zeta_{i}\right)>1$ 


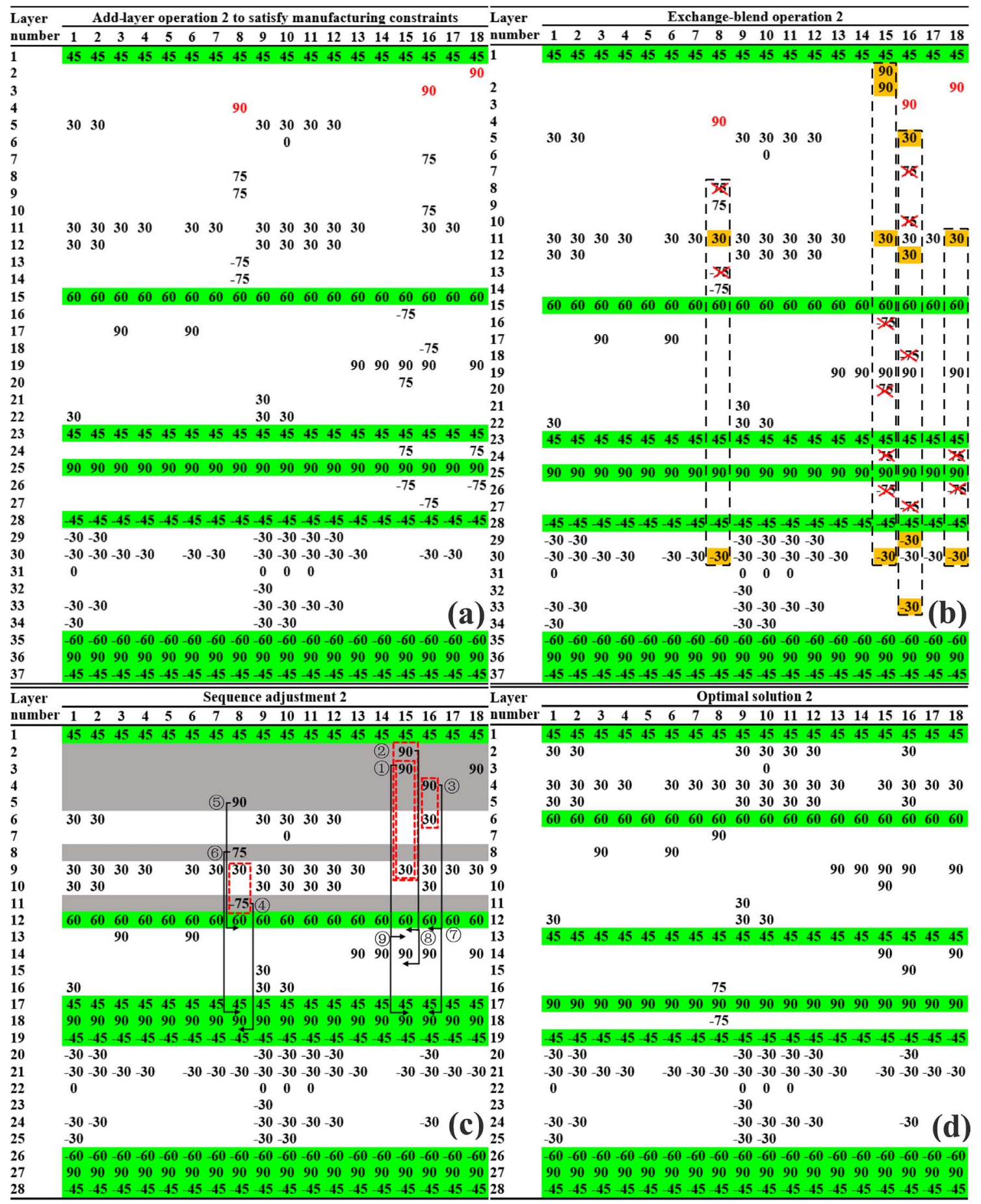

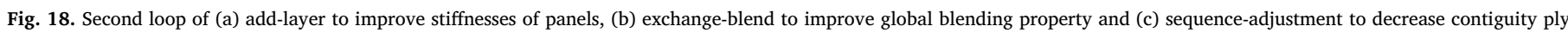
drops; (d) Optimal solution 2.

Algorithm 4: Sequence-adjustment operation

Success_sign $2=$ Success_sign $2+1$

Save the stacking sequence matrix $\Omega$; end if

end for

if Success_sign $2==$ length $\left(\overline{\boldsymbol{u}}_{s_{k}}\right)$

adjust_sign $=1$;

Obtain the total number of contiguity ply drops of the shared

layer $\omega_{r_{k}}^{k}$;

$k=k+1$
Algorithm 4: Sequence-adjustment operation

$$
\begin{aligned}
& \text { else } \\
& \text { adjust_sign }=0 \text {; } \\
& \text { end if }
\end{aligned}
$$

end for

if adjust_sign $==1$

Choose the best inserted stacking position with a minimum total number of contiguity ply drops, then update the stacking sequence matrix $\boldsymbol{\Omega}$ and its corresponding $\lambda_{c b}$, finally add the insert stacking position to the array ban_num; else 
Table 2

Variation of buckling load factors with corresponding thicknesses.

\begin{tabular}{ccccccc}
\hline \multirow{2}{*}{$\begin{array}{c}\text { Panel } \\
i\end{array}$} & \multicolumn{2}{c}{ Fig.16(c) } & \multicolumn{2}{c}{$\begin{array}{c}\text { Fig.17(d) } \\
\text { Solution } 1\end{array}$} & \multicolumn{2}{c}{ Fig.18(d) } \\
\cline { 2 - 7 } & $\lambda_{c b}\left(\zeta_{i}\right)$ & $N_{i}$ & $\lambda_{c b}\left(\zeta_{i}\right)$ & $N_{i}$ & $\lambda_{c b}\left(\zeta_{i}\right)$ & $N_{i}$ \\
\hline 1 & 0.9435 & 32 & 1.1315 & 34 & 1.1315 & 34 \\
2 & 1.0064 & 28 & 1.0064 & 28 & 1.0064 & 28 \\
3 & 0.9360 & 20 & 1.1182 & 22 & 1.1182 & 22 \\
4 & 0.9774 & 18 & 1.1925 & 20 & 1.1925 & 20 \\
5 & 1.1090 & 16 & 1.1090 & 16 & 1.1090 & 16 \\
6 & 1.1314 & 22 & 1.0049 & 22 & 1.0049 & 22 \\
7 & 0.9467 & 18 & 1.1551 & 20 & 1.1551 & 20 \\
8 & 1.0000 & 24 & 1.0000 & 24 & 1.1505 & 26 \\
9 & 1.0183 & 38 & 1.0239 & 38 & 1.0239 & 38 \\
10 & 0.9268 & 34 & 1.0959 & 36 & 1.0959 & 36 \\
11 & 1.0614 & 30 & 1.0627 & 30 & 1.0627 & 30 \\
12 & 1.0021 & 28 & 1.0021 & 28 & 1.0021 & 28 \\
13 & 1.1843 & 22 & 1.0520 & 22 & 1.0520 & 22 \\
14 & 1.0367 & 18 & 1.0349 & 18 & 1.0349 & 18 \\
15 & 0.9694 & 24 & 1.2270 & 26 & 1.1193 & 26 \\
16 & 1.0670 & 30 & 1.0005 & 30 & 1.0326 & 32 \\
17 & 0.9601 & 18 & 1.1715 & 20 & 1.1715 & 20 \\
18 & 1.0261 & 22 & 1.0116 & 22 & 1.2007 & 24 \\
\hline Total layers & 442 & \multicolumn{6}{c}{456} & 462 \\
\hline$W$ (kg) & 27.6439 & 28.4444 & 28.7236 \\
\hline
\end{tabular}

Algorithm 4: Sequence-adjustment operation

$k=k+1 ;$
end if
end if
end while

\section{Enhanced design framework}

Based on the GBM and the above three operations, the design framework presented in Part I of this work is simplified and enhanced, as shown in Fig. 14. At the global level [23], the initial five steps are the same with that of Part I of this work: (i) the MSPT, (ii) prediction of minimum thickness distribution, (iii) layer shape prediction, (iv) ply orientation identification and (v) stacking sequence optimization. Subsequently, at the local level, the add-layer, exchange-blend and sequence-adjustment operations are revised and improved, with the GBM used in the first two operations for identifying the best stacking positions of layers to maximize the global blending property. The enhanced tapered-composite-structure design framework is named as TCSDF.

As compared to the original design framework, the GSLB method and stacking-sequence optimization procedure are performed only once in the design process. In Fig. 14, at the global level, the shapes, ply orientations of shared layers and stacking sequence are identified, in which constraints CT1, CT2, СT8 and CT9 are satisfied. Then at the local level, the add-layer, exchange-blend and sequence-adjustment operations are performed twice: in the first loop, the panels that violate buckling constraint are selected for add-layer operation, and constraints CT3 to CT6 are satisfied in exchange-blend and sequence-adjustment operations. However, after the first loop, some panels may violate the ply orientation mismatch constraint CT7, thus, in the second loop, layers are added to these panels by add-layer operation and the blending property is further improved by exchange-blend and sequence-adjustment operations.

Furthermore, the more manufacturing constraints are served, the heavier structure is anticipated. Therefore, layers are added for different reasons: the buckling constraint or the manufacturing constraints. The final optimal solution is a trade-off between constraints and weight, which is controlled by the designer. In this work, we try to achieve an perfectly blended optimal solution that can fully satisfy all manufacturing constraints.

\section{Numerical results}

The TCSDF is implemented for the 18-panel problem [23] (see Fig. 15(a)), in which panels are symmetrical and balanced laminates with even ply number. Candidate ply orientations are set as $\left\{0^{\circ}, \pm 15^{\circ}, \pm 30^{\circ}, \pm 45^{\circ}, \pm 60^{\circ}, \pm 75^{\circ}, 90^{\circ}\right\}$, see Fig. 15(b). The material is graphite/epoxy IM7/8552 with properties $E_{1}=141 \mathrm{GPa}(20.5$ $\mathrm{Msi}), E_{2}=9.03 \mathrm{GPa}(1.31 \mathrm{Msi}), G_{12}=4.27 \mathrm{GPa}(0.62 \mathrm{Msi}), \nu_{12}=0.32$ and ply thickness is $t=0.191 \mathrm{~mm}(0.0075 \mathrm{in})$. In the design results given in Figs. 16-18, the elements corresponding to -1 in the stacking sequence matrix $\boldsymbol{\Omega}$ are deleted. The detailed design process please refer the Supplementary Material. The main design procedures and results are summarized as follows.

The first five steps of the TCSDF are the same with that of Part I and the design results are summarized in Fig. 16. Fig. 16(a) presents the minimum thickness distribution of the structure by setting the best ply orientation of each panel in individual panel, where the balance requirement is satisfied. Fig. 16(b) gives the stacking sequence after shape and ply orientation identification. Fig. 16(c) is the result of stacking sequence optimization. The constraints CT1 to CT4, СT8 and СT9 are satisfied in Fig. 16(c), however, to obtain the lightweight structure, some panels violate buckling constraint, as shown in Table 2 (values in red). Therefore, the add-layer, exchange-blend and sequence adjustment operations are performed in terms of the GBM to design the structure. Results are given in Fig. 17: in Fig. 17(a), layers (values in red) are added as individual layers or blended with the original shared layer in panels 1, 3, 4, 7, 10, 15 and 17 respectively. Subsequently, in Fig. 17(b), some layers (red crosses) are exchanged and blended (the layers in orange) to big shared layers in panels 3, 4, 6, 7, 13, 16 and 17 (in the black dashed box), respectively. Finally, in Fig. 17(c), due to the increase of buckling load factors in some panels, the stacking sequence is adjusted by inserting small shared layers (rows in grey) between bigger ones considering the manufacturing constraints CT3 to CT6 (layers in the red dashed box violate CT4). The optimal solution 1 is given in Fig. 17(d) with corresponding cross-sections in Fig. 19(a) and (c), and buckling load factors and number of plies in Table 2. However, solution 1 cannot satisfy constraints CT5 to CT7, since the ply orientations of panels 8,16 and 18 are limited by buckling load factors (see Table 2), results in lots of ply orientation mismatches between adjacent panels $\{7,8\},\{12,16\}$ and $\{17,18\}$ respectively, see Fig. 19(c). Accordingly, based on the TCSDF, layers are added to panels 8, 16 and 18: the operations of add-layer, exchange-blend and sequence adjustment are implemented once more, results are given in Fig. 18. The optimal solution 2 is shown in Fig. 18(d) with corresponding crosssections in Fig. 19(b) and (d), and the buckling load factors and numbers of plies in Table 2. In Fig. 19, the blending property of solution 2 is apparently improved compared to that of solution 1, especially between panels $\{7,8\},\{12,16\}$ and $\{17,18\}$. All manufacturing constraints are satisfied in solution 2: there is only one position with two $(D N=2)$ contiguity ply drops (see Fig. 19(b)), and all other contiguity ply-drop numbers between adjacent panels are equal to 1 . 


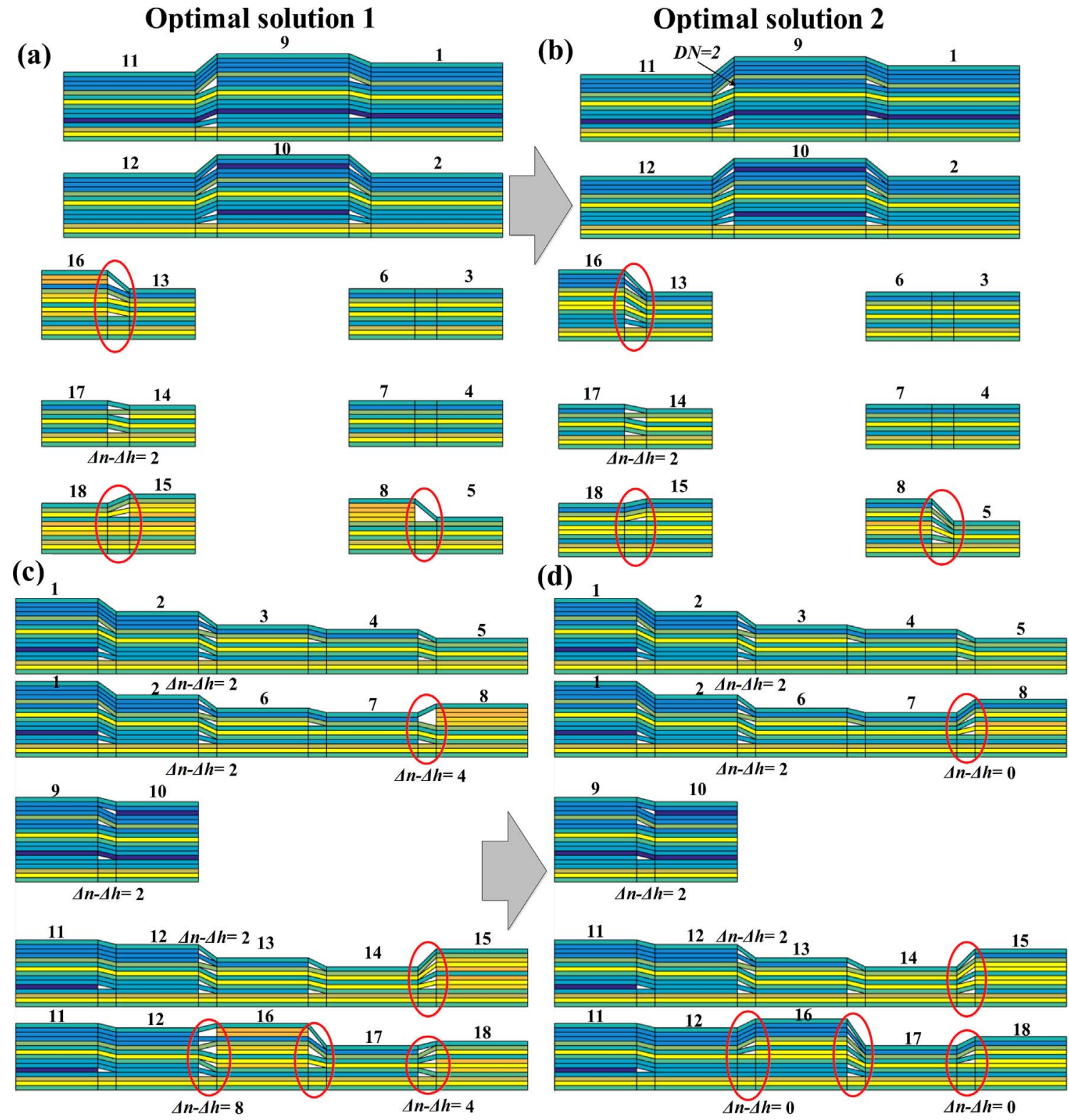

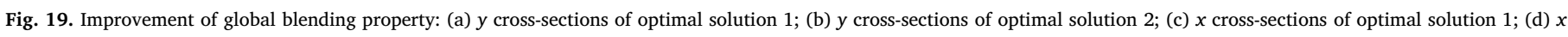
cross-sections of optimal solution 2 .

To demonstrate the important effect of the GBM applied in the design process, the variation of ply-panel continuity ratio is depicted in Fig. 20: here green lines denote the add-layer operation with each point representing the ply-panel continuity ratio after a layer is added. Since the layers are added as individual layers or blend to original shared layers, the ply-panel continuity ratio decreases due to the thickness increase in panels in the add-layer operations. The red lines denote the exchange-blend operation with each point representing the ply-panel continuity ratio after layers are exchanged and blended. Since layers were deleted, exchanged and finally blended to big shared layers without thickness variation, the ply-panel continuity ratio increases in the exchange-blend operation. Note that, each point in Fig. 20 is chosen from several candidate points with maximum ply-panel continuity ratio, the eliminated points are not drawn in Fig. 20.

The final optimal solution 2 is compared with the results obtained by other researchers in the last 3 years (in Table 3), in which the total ply number of each panel is even. In Table 3, the weight of the present solution is the minimum and it perfectly satisfies manufacturing constraints introduced in this work. The blending property of the four solutions can be checked in Fig. 21. Their corresponding ply-panel continuity ratios are evaluated in Table 3 , it is worth mentioning that similar ply-panel continuity ratios of different optimal results are acceptable, that is to say the blending property of the four solutions are very good. The present solution reaches the maximum global blending property in terms of the present thickness distribution of multiple ply orientations, which can be verified by the GSLB method [21]. Hence, the GBM is an efficient tool to guide the design for maximizing blending property. Incorporating with the GBM, the TCSDF is simplified and its computational cost is reduced significantly. For the 18-panel problem, it needs no more than $1 \mathrm{~s}$ using Matlab 2016b on a desktop computer 


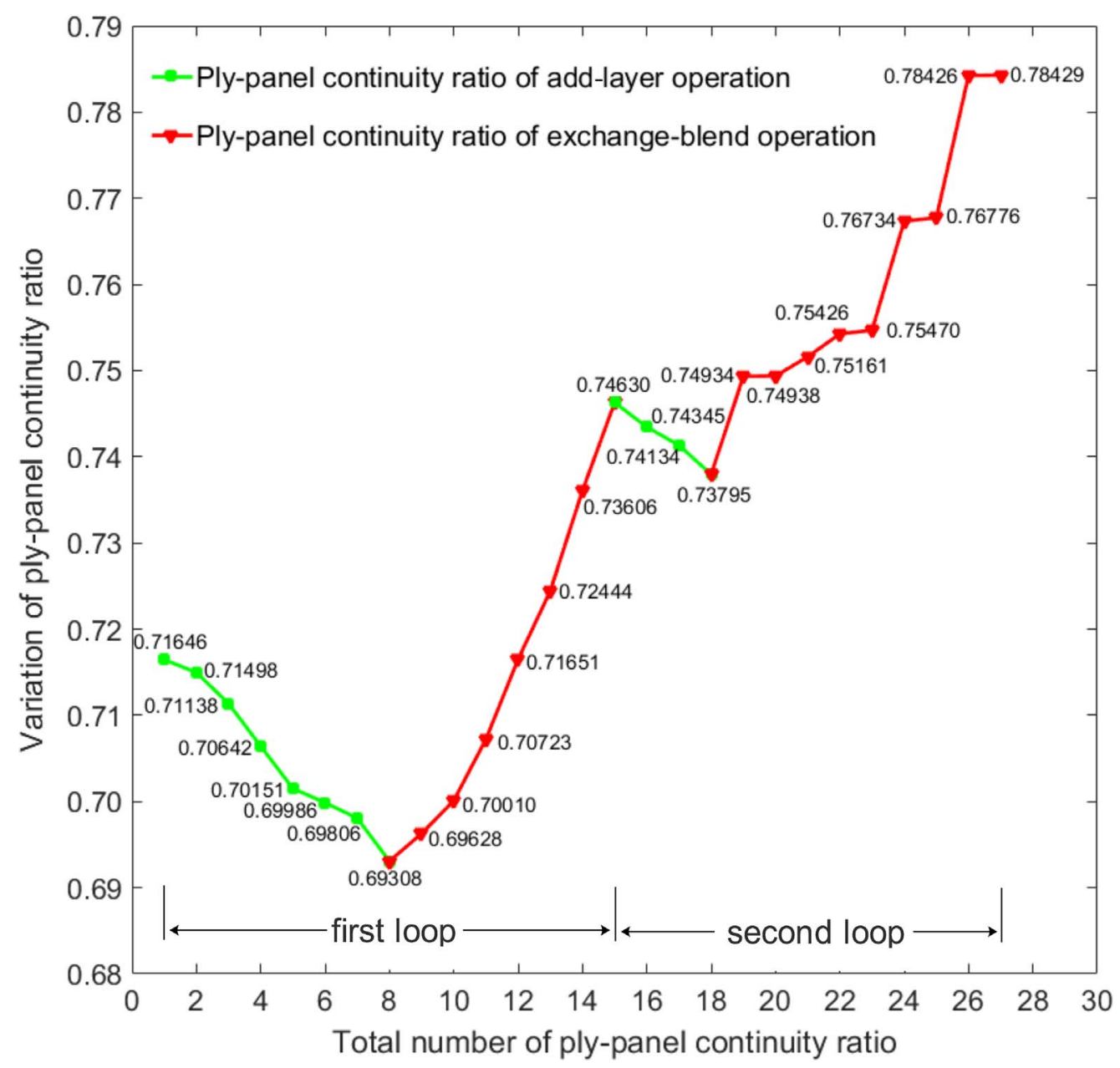

Fig. 20. Variation of ply-panel continuity ratio in design process.

Table 3

Comparison of different optimal solutions: ply numbers, weights and ply-panel continuity ratios.

\begin{tabular}{lllll}
\hline Panel & Fan $[24]$ & Yang $[25]$ & Irisarri $[26]$ & Present solution \\
\hline 1 & 34 & 34 & 34 & 34 \\
2 & 28 & 28 & 30 & 28 \\
3 & 24 & 22 & 22 & 22 \\
4 & 20 & 20 & 18 & 20 \\
5 & 16 & 16 & 18 & 16 \\
6 & 24 & 22 & 22 & 22 \\
7 & 20 & 20 & 18 & 20 \\
8 & 26 & 26 & 26 & 26 \\
9 & 38 & 38 & 38 & 38 \\
10 & 36 & 36 & 38 & 36 \\
11 & 30 & 30 & 30 & 28 \\
12 & 28 & 28 & 30 & 22 \\
13 & 22 & 22 & 22 & 18 \\
14 & 20 & 20 & 18 & 32 \\
15 & 26 & 26 & 26 & 20 \\
16 & 32 & 32 & 30 & 24 \\
17 & 20 & 20 & 18 & 462 \\
18 & 24 & 24 & 22 & 28.7236 \\
\hline Total layers & 468 & 464 & 460 & 0.7843 \\
\hline$W(\mathrm{~kg})$ & 29.0028 & 28.8167 & 28.8539 & \\
\hline C & 0.7767 & 0.8174 & 0.8003 & \\
\hline
\end{tabular}

with an i7-6700 K CPU ( 4 cores, $4.00 \mathrm{GHz}$ ) and a $32 \mathrm{~GB}$ RAM to find the optimal solution.

\section{Conclusions}

In this work, a mathematical model for the optimization of tapered composite structures is constructed. The objective is to minimize the weight accounting for the buckling and manufacturing constraints, in which several new constraints for ply drops and their corresponding detection techniques are introduced. Based on the framework proposed in Part I of this work, to improve the design flexibility and feasibility, characterize the global blending property and guide the design process, a global blending model (GBM) is proposed to address the problems of addition, deletion and blending of layers, which is a "sensitive parameter" for the global blending property. The new tapered composite structures design framework is incorporated with the GBM, in which the add-layer, exchange-blend and sequence-adjustment operations are revised and enhanced, consequently, reducing the computational cost of the design process and improving the solution's blending quality. An 18-panel benchmark problem is adopted to verify the TCSDF. The obtained lightest solution which satisfies manufacturing constraints indicates that it outperformed previous heuristic algorithms with a higher efficiency. In particular, the GBM can be used as an index to characterize the global blending property or as a tool to guide the maximum blending design of tapered composite structures. Future work should 

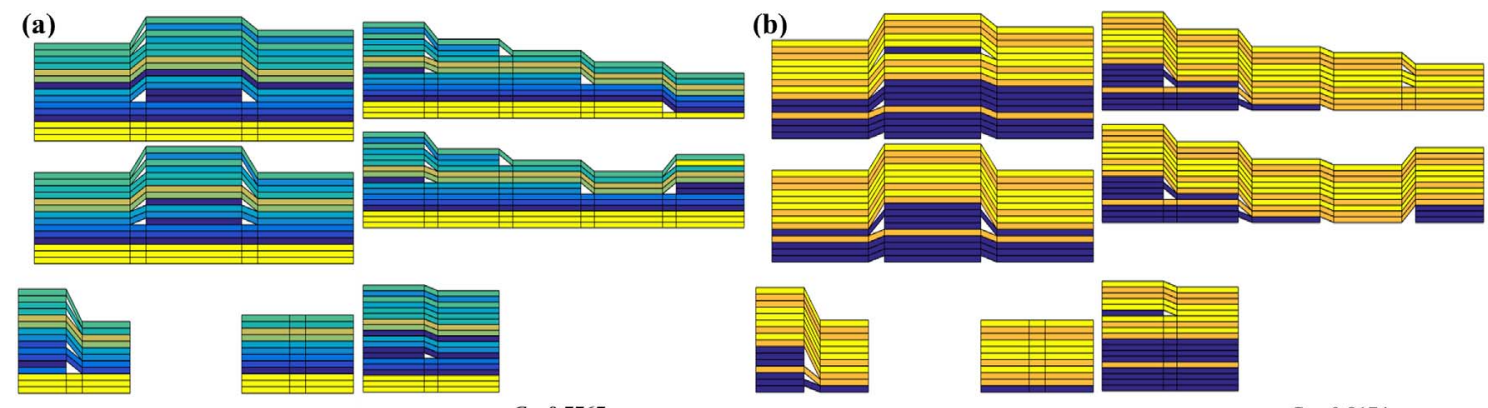

\section{$C=0.7767$}
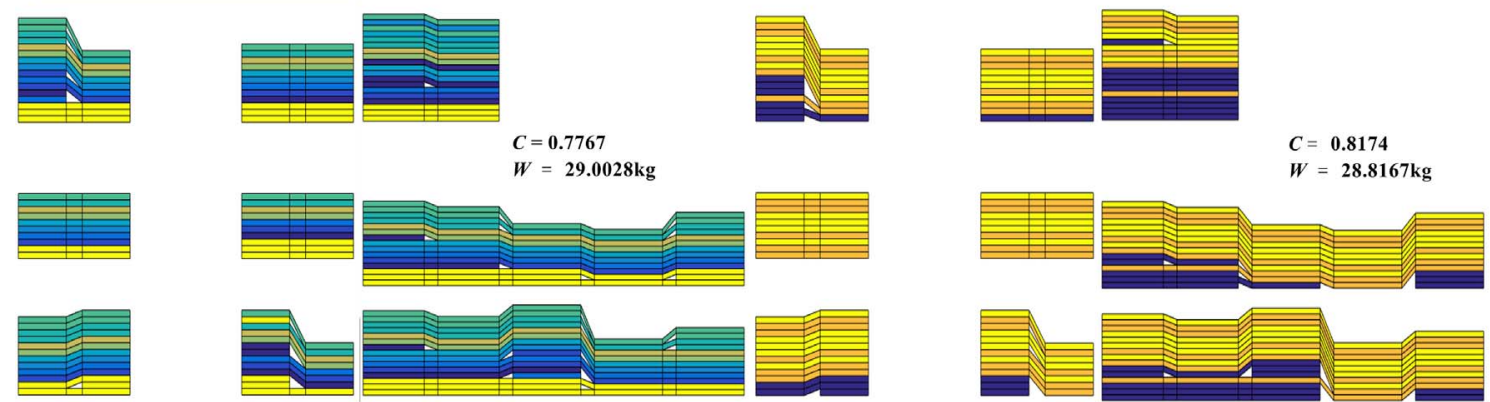

(c)
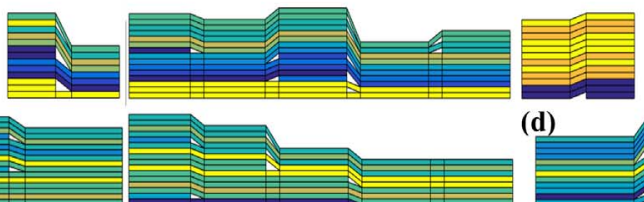

(d)
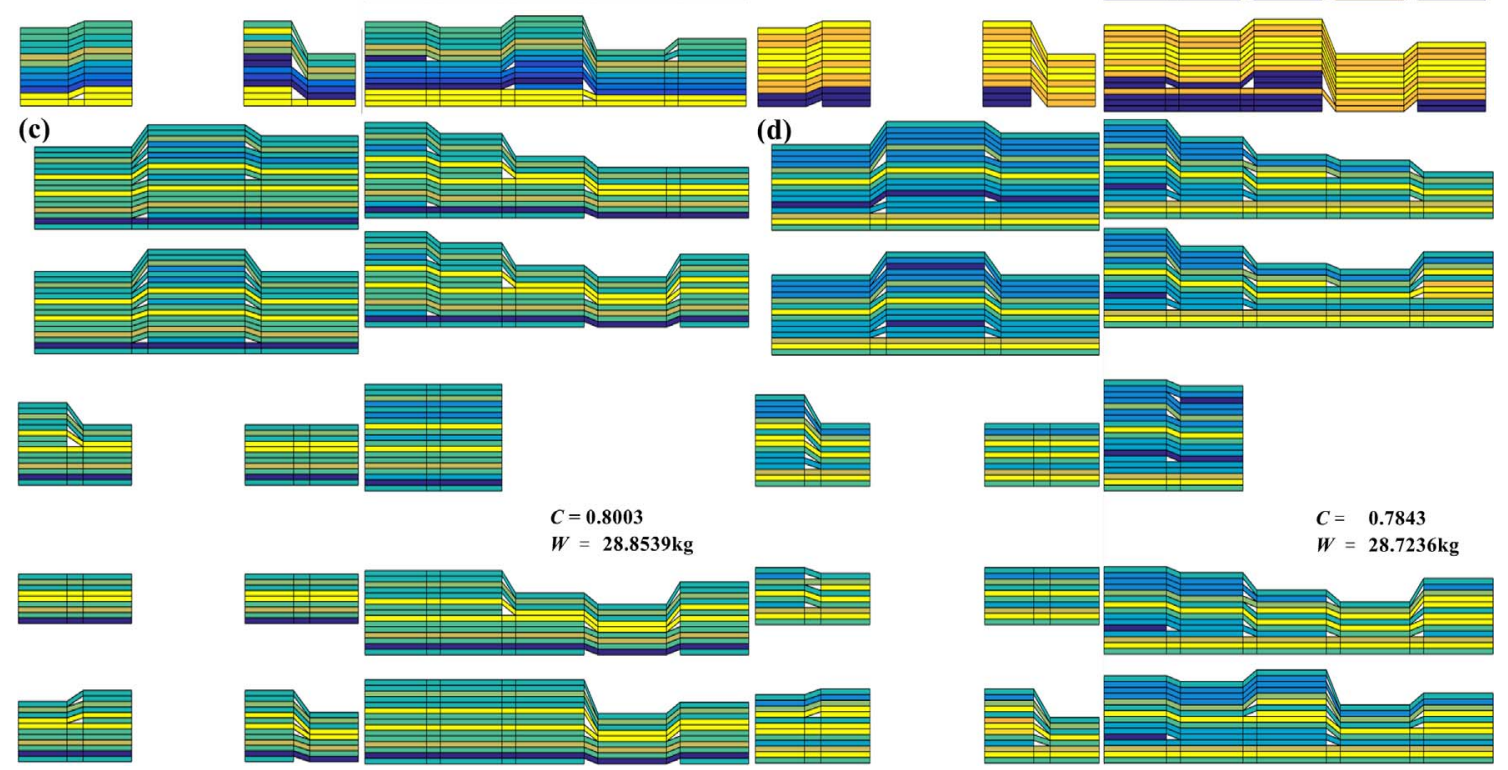

Fig. 21. Blending property comparison of different solutions: (a) Fan [24]; (b) Yang [25]; (c) Irisarri [26]; (d) Present solution.

focus on introduction of other mechanical requirements such as strength and strain constraints in the TCSDF, and efforts should be made to formulate the design procedures mathematically.

\section{Acknowledgements}

This work is supported by the National Natural Science Foundation of China (Nos. 11572134, 51375386) and China Postdoctoral Science Foundation funded project (No. 2017M612443).

\section{Appendix A}

In the manufacturing constraints, some are imposed implicitly in the design process, for instance: the symmetry (CT1), covering (CT8) and continuity layers (CT9). However, the other constraints are detected via special developed computer programs. The procedures to detect the manufacturing constraints CT2-CT7 are introduced as follows, in which parameters are predefined in Section 2.1.

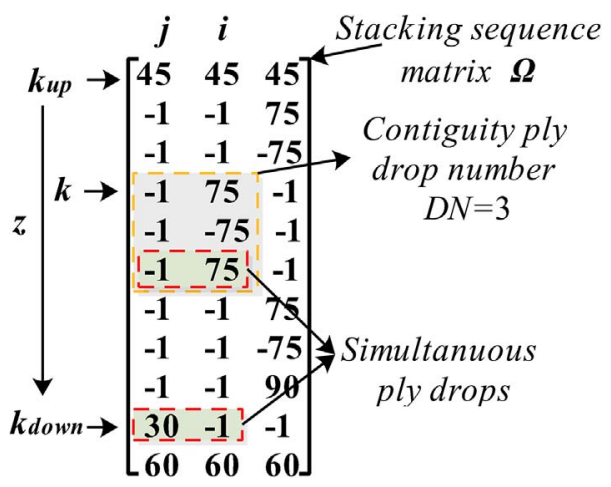

Fig. A1. Detection of contiguity and simultaneous ply drops in stacking sequence matrix $\boldsymbol{\Omega}$. 
CT2. Balance. Balance constraint is shown in Eq. (A.1), if $n_{j}^{\theta_{r}} \neq n_{j}^{-\theta_{r}}$, the ply numbers of $n_{j}^{\theta_{r}}$ and $n_{j}^{-\theta_{r}}$ should be adjusted in the stacking sequence $\zeta_{j}(j=1,2, \ldots, P)$.

$n_{j}^{\theta_{r}}=n_{j}^{-\theta_{r}}, \theta_{r} \neq 0^{\circ}$ or $90^{\circ}, \quad j=1,2, \ldots, P, \quad r=1,2, \ldots, M$

CT3. Contiguity. A variable $\mathrm{Num}_{j}$ is used in Eq. (A.2) to represent the number of successive layers with the same ply orientation in stacking sequence $\zeta_{j}(j=1,2, \ldots, P)$. The number of plies in panel $j$ is $n_{j}$, and the constraint is detected by the procedure in Eq. (A.2): if vio_CT3 $\neq 0$, the constraint CT3 is violated. Similarly, for the following constraints, the vio_CTi has the same meaning.

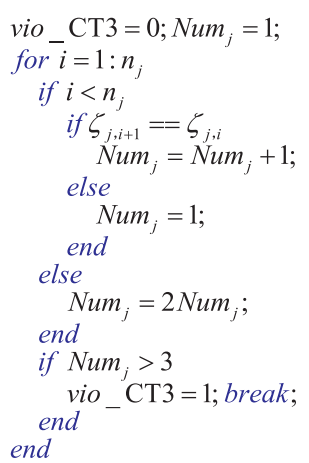

CT4. Disorientation. The disorientation constraint is detected via Eq. (A.3).

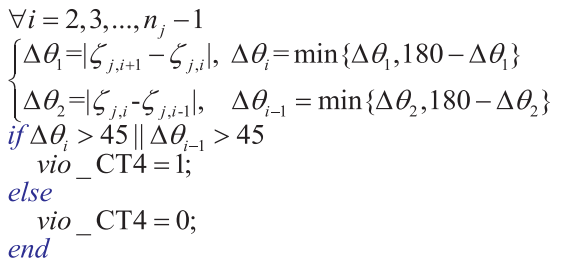

CT5. Contiguity ply-drop number limit $D N \leq 2$. Since every shared layer is in a row in the stacking sequence matrix, it is convenient to detect the contiguity ply drop numbers for any two adjacent panels $i$ and $j$, the numbers are detected via the procedure presented in Eq. (A.4). It assumes that the current shared layer row number is $k, i$ is the current panel, $j$ is the adjacent panel of panel $i$. As shown in Fig. A.1, contiguity ply-drop number is detected in a yellow dashed box, where $k_{u p}$ and $k_{\text {down }}$ are the stacking positions above and below the ply drop respectively.

CT6. Simultaneous ply-drop limit. This constraint can be detected via Eq. (A.5), and it is detected in a red dashed box in Fig. A.1, where $k_{u p}$ and $k_{\text {down }}$ are the same with those of Eq. (A.4).

vio_CT5 $=0$; vio_CT $6=0$;

$\%$ find the stacking position up and down of the drop position

for $z=1: k$

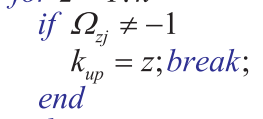

end

for $z=k+1: Q$

if $\Omega_{z j} \neq-1$

$k_{\text {down }}=z ;$ break;

end

$\%$ check the contiguity ply-drop number limit

for $z=k_{\text {up }}: k_{\text {down }}$

if $\Omega_{z j}=-1 \& \& \Omega_{z i} \neq-1$

$D N=D N+1$

end

if $D N>2$

vio_CT5 = 1; break;

end

$\%$ check the simultaneous ply-drop limit

if $\left(\Omega_{k_{u p} j} \neq-1 \& \& \Omega_{k_{u p} i}==-1\right) \|\left(\Omega_{k_{\text {down }} j} \neq-1 \& \& \Omega_{k_{\text {down }} i}==-1\right)$

vio_CT6 =1;

end

CT.7 Ply orientation mismatch limit $\Delta n-\Delta h \leq 2$. This constraint is detected by Eq. (A.6). 
vio $\mathrm{CT} 7=0$

$\Delta n=0 ; \quad \%$ total ply drop numbers between adjacnet panels $i$ and $j$

$\Delta h=\left|n_{i}-n_{j}\right| ; \%$ thickness difference between adjacnet panels $i$ and $j$

for $k=1: Q$

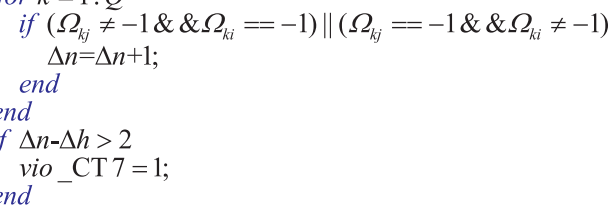

\section{Appendix B. Supplementary data}

Supplementary data associated with this article can be found, in the online version, at http://dx.doi.org/10.1016/j.compstruct.2017.11.062.

\section{Reference}

[1] He K, Hoa SV, Ganesan R. The study of tapered laminated composite structures: a review. Compos Sci Tech 2000;60:2643-57.

[2] Kristinsdottir BP, Zabinsky ZB, Tuttle ME, Neogi S. Optimal design of large composite panels with varying loads. Compos Struct 2001;51(1):93-102.

[3] Liu BY, Haftka RT. Composite wing structural design optimization with continuity constraints. In: 42nd AIAA/ASME/ASCE/AHS/ASC Structures, Structural Dynamics, and Materials Conference, Seattle, WA, 16-19 April, 2001.

[4] Van Campen JMJF, Seresta O, Abdalla MM, Gürdal Z. General blending definitions for stacking sequence design of composite laminate structures. In: Proceedings of 49th AIAA/ASME/ASCE/AHS/ASC structures, structural dynamics, and materials conference, Schaumburg, IL, USA; 7-10 April 2008.

[5] Liu WL, Richard B. Optimum Buckling Design of Composite Wing Cover Panels with Manufacturing Constraints. In: 48th AIAA/ASME/ASCE/AHS/ASC Structures, Structural Dynamics, and Materials Conference, Honolulu, Hawali, 23-26 April 2007.

[6] Seresta O, Gürdal Z, Adams DB, Watson LT. Optimal design of composite wing structures with blended laminates. Compos B: Eng 2007;38:469-80.

[7] Liu DZ, Toropov VV, Querin OM, Barton DC. Bi-level optimization of blended composite panels. In: 50th AIAA/ASME/ASCE/AHS/ASC Structures, Structural Dynamics, and Materials Conference, Palm Springs, California, 4-7 May, 2009.

[8] Soremekun G, Gürdal Z, Kassapoglou C, Toni D. Stacking sequence blending of multiple composite laminates using genetic algorithms. Compos Struct 2002;56(1):53-62.

[9] Adams DB, Watson LT, Gürdal Z, Anderson-Cook CM. Genetic algorithm optimization and blending of composite laminates by locally reducing laminate thickness. Adv Eng Softw 2004;35(1):35-43.

[10] Adams DB, Watson LT, Setoodeh O, Gürdal Z. Global/Local iteration for blended composite laminate panel structure optimization subproblems. Mech Adv Materi Struct 2007;14(2):139-50.

[11] Seresta O, Abdalla MM, Gürdal Z. A genetic algorithm based blending scheme for design of multiple composite laminates. In: Proceedings of 50th AIAA/ ASME/ ASCE/AHS/ASC structures, structural dynamics, and materials conference, Palm Springs, California, USA; 4-7 May 2009.

[12] IJsselmuiden ST, Abdalla MM, Seresta O, Gürdal Z. Multi-step blended stacking sequence design of panel assemblies with buckling constraints. Compos B: Eng 2009;40:329-36.

[13] Bruyneel M. SFP - A new parameterization based on shape functions for optimal material selection: application to conventional composite plies. Struct Multidiscipl Optim 2011;43:17-27.

[14] Panesar AS, Weaver PM. Optimisation of blended bistable laminates for a morphing flap. Compos Struct 2012;94:3092-105.
[15] Graeme JK, Martins JRRA. A laminate parametrization technique for discrete plyangle problems with manufacturing constraints. Struct Multidiscipl Optim. 2013;48:379-93.

[16] Zein S, Colson B, Grihon S. A primal-dual backtracking optimization method for blended composite structures. Struct Multidisc Optim 2012;45:669-80.

[17] Zein S, Basso P, Grihon S. A constraint satisfaction programming approach for computing manufacturable stacking sequences. Comput Struct 2014;136:56-63.

[18] Zein S, Bruyneel M. A bilevel integer programming method for blended composite structures. Adv. Eng Software 2015;79:1-12.

[19] Zhou M, Fleury R, Kemp M. Optimization of composite: Recent advances and application. In: The 7th Altair CAE Technology Conference, Altair; 2011.

[20] Jing Z, Fan XL, Sun Q. Stacking sequence optimization of composite laminates for maximum buckling load using permutation search algorithm. Compos Struct 2015;121:225-36.

[21] Jing Z, Fan XL, Sun Q. Global shared layer blending method for stacking sequence optimization design and blending of composite structures. Compos B: Eng. 2015;69:181-90.

[22] Jing Z, Sun Q, Silberschmidt VV. Sequential permutation table method for optimization of stacking sequence in composite laminates. Compos Struct 2016;141:240-52.

[23] Jing Z, Sun Q, Silberschmidt VV. A framework for design and optimization of tapered composite structures Part I: From individual panel to global blending structure. Compos Struct 2016;154:106-28.

[24] Fan HT, Wang H, Chen XH. An optimization method for composite structures with ply-drops. Compos Struct 2016;136:650-61.

[25] Yang JB, Song BF, Zhong XP, Jin P. Optimal design of blended composite laminate structures using ply drop sequence. Compos Struct 2016;135:30-7.

[26] Irisarri F-X, Lasseigne A, Leroy FH, Le Riche R. Optimal design of laminated composite structures with ply drops using stacking sequence tables. Compos Struct 2014;17:559-69.

[27] Macquart T, Bordogna MT, Lancelot P, Breuker RD. Derivation and application of blending constraints in lamination parameter space for composite optimisation. Compos Struct 2016;135:224-35.

[28] Zehnder N, Ermanni P. A methodology for the global optimization of laminated composite structures. Compos Struct 2006;72(3):311-20.

[29] Giger M, Keller D, Ermanni P. A graph-based parameterization concept for global laminate optimization. Struct Multidiscipl Optim 2008;36:289-305.

[30] Kim J-S, Kim C-G, Hong C-S. Practical design of tapered composite structures using the manufacturing cost concept. Compos Struct 2001;51(3):285-99.

[31] Kim J-S, Kim C-G, Hong C-S. Optimum design of composite structures with ply drop using genetic algorithm and expert system shell. Compos Struct 1999;46(2):171-87.

[32] Allaire G, Delgado G. Stacking sequence and shape optimization of laminated composite plates via a level-set method. J Mech Phys Solids 2016;97:168-96. 